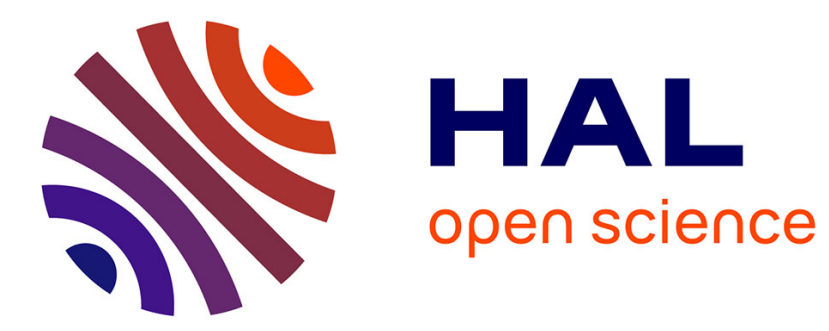

\title{
Diurnal variations of Titan's ionosphere
}

J. Cui, M. Galand, R. V. Yelle, Veronique Vuitton, J.-E. Wahlund, Panayotis Lavvas, I. C. F. Müller-Wodarg, T. E. Cravens, W. T. Kasprzak, J. H. Waite Jr.

\section{- To cite this version:}

J. Cui, M. Galand, R. V. Yelle, Veronique Vuitton, J.-E. Wahlund, et al.. Diurnal variations of Titan's ionosphere. Journal of Geophysical Research Space Physics, 2009, 114 (6), pp.A06310. 10.1029/2009JA014228 . insu-00501501

\section{HAL Id: insu-00501501 https://hal-insu.archives-ouvertes.fr/insu-00501501}

Submitted on 5 Mar 2021

HAL is a multi-disciplinary open access archive for the deposit and dissemination of scientific research documents, whether they are published or not. The documents may come from teaching and research institutions in France or abroad, or from public or private research centers.
L'archive ouverte pluridisciplinaire HAL, est destinée au dépôt et à la diffusion de documents scientifiques de niveau recherche, publiés ou non, émanant des établissements d'enseignement et de recherche français ou étrangers, des laboratoires publics ou privés. 


\title{
Diurnal variations of Titan's ionosphere
}

\author{
J. Cui, ${ }^{1}$ M. Galand, ${ }^{1}$ R. V. Yelle, ${ }^{2}$ V. Vuitton, ${ }^{3}$ J.-E. Wahlund, ${ }^{4}$ P. P. Lavvas, ${ }^{2}$ \\ I. C. F. Müller-Wodarg, ${ }^{1}$ T. E. Cravens, ${ }^{5}$ W. T. Kasprzak, ${ }^{6}$ and J. H. Waite Jr. ${ }^{7}$ \\ Received 12 March 2009; revised 24 April 2009; accepted 29 April 2009; published 18 June 2009.
}

[1] We present our analysis of the diurnal variations of Titan's ionosphere (between 1000 and $1300 \mathrm{~km}$ ) based on a sample of Ion Neutral Mass Spectrometer (INMS) measurements in the Open Source Ion (OSI) mode obtained from eight close encounters of the Cassini spacecraft with Titan. Although there is an overall ion depletion well beyond the terminator, the ion content on Titan's nightside is still appreciable, with a density plateau of $\sim 700 \mathrm{~cm}^{-3}$ below $\sim 1300 \mathrm{~km}$. Such a plateau is a combined result of significant depletion of light ions and modest depletion of heavy ones on Titan's nightside. We propose that the distinctions between the diurnal variations of light and heavy ions are associated with their different chemical loss pathways, with the former primarily through "fast" ion-neutral chemistry and the latter through "slow" electron dissociative recombination. The strong correlation between the observed night-to-day ion density ratios and the associated ion lifetimes suggests a scenario in which the ions created on Titan's dayside may survive well to the nightside. The observed asymmetry between the dawn and dusk ion density profiles also supports such an interpretation. We construct a time-dependent ion chemistry model to investigate the effect of ion survival associated with solid body rotation alone as well as superrotating horizontal winds. For long-lived ions, the predicted diurnal variations have similar general characteristics to those observed. However, for short-lived ions, the model densities on the nightside are significantly lower than the observed values. This implies that electron precipitation from Saturn's magnetosphere may be an additional and important contributor to the densities of the short-lived ions observed on Titan's nightside.

Citation: Cui, J., M. Galand, R. V. Yelle, V. Vuitton, J.-E. Wahlund, P. P. Lavvas, I. C. F. Müller-Wodarg, T. E. Cravens, W. T. Kasprzak, and J. H. Waite Jr. (2009), Diurnal variations of Titan's ionosphere, J. Geophys. Res., 114, A06310, doi:10.1029/2009JA014228.

\section{Introduction}

[2] Titan, the largest moon of Saturn, has a substantial ionosphere that was first detected on the basis of the dualfrequency Doppler data obtained with the Voyager 1 radio occultation experiment [Bird et al., 1997]. The discovery was later confirmed by in-situ measurements of the electron distribution made by the Cassini Radio and Plasma Wave Science (RPWS) instrument [Wahlund et al., 2005] as well

\footnotetext{
${ }^{1}$ Space and Atmospheric Physics Group, Department of Physics, Imperial College, London, UK.

${ }^{2}$ Lunar and Planetary Laboratory, University of Arizona, Tucson, Arizona, USA.

${ }^{3}$ Laboratoire de Planétologie de Grenoble, Université Joseph Fourier/ CNRS, Grenoble, France.

${ }^{4}$ Uppsala Division, Swedish Institute of Space Physics, Uppsala, Sweden.

${ }^{5}$ Department of Physics and Astronomy, University of Kansas, Lawrence, Kansas, USA.

${ }^{6}$ Solar System Exploration Division, NASA Goddard Space Flight Center, Greenbelt, Maryland, USA.

${ }^{7}$ Space Science and Engineering Division, Southwest Research Institute, San Antonio, Texas, USA.

Copyright 2009 by the American Geophysical Union. 0148-0227/09/2009JA014228
}

as the radio occultation results from the Cassini Radio Science (RSS) instrument [Kliore et al., 2008].

[3] Complementary to the RPWS and RSS investigations of Titan's ionospheric electrons, in-situ measurements of the abundances of various ion species have been made by the Cassini Ion Neutral Mass Spectrometer (INMS) in the Open Source Ion (OSI) mode [Cravens et al., 2005]. Previous studies based on the INMS ion data revealed that the composition of Titan's ionosphere is very complex, with $\sim 50$ ion species above the detection threshold [Cravens et al., 2005, 2006; Vuitton et al., 2006, 2007; Waite et al., 2007]. A typical ion mass spectrum obtained with the INMS shows distinct ion groups that are separated in mass-tocharge ratio (hereafter $M / Z$ ) by $\sim 12$ Da [Cravens et al., 2006; Vuitton et al., 2007]. In addition, analyses of the data obtained with the Ion Beam Spectrometer (IBS) and the Electron Spectrometer (ELS) of the Cassini Plasma Spectrometer (CAPS) also revealed the presence of heavier positive ions with $M / Z \sim 130,170$ and 335 [Waite et al., 2007], as well as negative ions in $M / Z$ groups of $10-30$, $30-50,50-80,80-110,110-200$ and $200+$ Da in Titan's ionosphere [Coates et al., 2007].

[4] The two main ionization sources of Titan's ionosphere are solar radiation in the EUV (and soft X-rays) and 
energetic particles from Saturn's magnetosphere, with their relative importances varying with local solar time on Titan as well as the location of the satellite with respect to Saturn's magnetosphere [e.g., Gan et al., 1992; Keller et al., 1992, 1994; Keller and Cravens, 1994; Roboz and Nagy, 1994; Fox and Yelle, 1997; Nagy and Cravens, 1998; Galand et al., 1999, 2006; Cravens et al., 2004, 2005, 2006, 2008a, 2008b; Agren et al., 2007, 2009; De La Haye et al., 2008]. Meteoritic ablation may also have some contributions to the ion production near $\sim 700 \mathrm{~km}[$ e.g., Ip, 1990; English et al., 1996; Molina-Cuberos et al., 2001], and cosmic ray ionization could be effective down to $\sim 65 \mathrm{~km}$ [e.g., López-Moreno et al., 2008]. Comparison of modeling output with a multi-instrumental data set (including the INMS ion and RPWS and CAPS electron data acquired during the first Titan flyby of Cassini, known in project parlance as TA) demonstrates that the solar EUV radiation is the dominant ion source on Titan's dayside [Galand et al., 2006]. This is further supported by the recent analysis of a large sample of the RPWS data, investigating the dependence of peak altitude and density of the observed electron distribution with solar illumination angle [Ägren et al., 2009]. Photoionization by solar EUV radiation may also be important well beyond the terminator, as a result of the extended nature of Titan's atmosphere [Müller-Wodarg et al., 2000; Cravens et al., 2004; Agren et al., 2009]. However, deep in Titan's nightside (as sampled by the Cassini T5 and T21 flybys), the primary ion source has been proposed to be precipitating electrons from Saturn's magnetosphere [Cravens et al., 2008b; Ågren et al., 2007, 2009]. Cravens et al. [2008a] also suggested that precipitation of energetic protons and oxygen ions from Saturn's magnetosphere may be important between 500 and $1000 \mathrm{~km}$, contributing to the ionospheric layer in the region of 500$600 \mathrm{~km}$ observed by the RSS experiment [Kliore et al., 2008].

[5] In this paper, we investigate further the source for Titan's nightside ionosphere, focusing on the observed diurnal variations of different ion species based on a large sample of INMS measurements in the OSI mode covering both the dayside and nightside of Titan. We show that the observed ion densities vary in a regular way that is tightly correlated with chemical lifetime. Electron precipitation and chemical survival of dayside ions, coupled with strong horizontal winds, are both investigated through comparison of models for these sources with the observations. These comparisons show that characteristics of both sources are seen in the data. Details of the INMS observations and data analysis are summarized in section 2 . We present our main results in section 3 , and discuss possible interpretations of the data in section 4 . In section 5, we compare the observed diurnal variations of Titan's ionosphere with results from a time-dependent ion chemistry model which also takes into account the effects of superrotating horizontal neutral winds. Finally, we summarize and conclude in section 6 .

\section{Observations and Data Analysis}

[6] Within the four years' length of the prime Cassini mission, there have been over 40 encounters with Titan. This work is based on the INMS data acquired during 8 of them, for which the measurements in the OSI mode are available (see section 2.2 for details). A complete description of the INMS instrument is given by Waite et al. [2004].

\subsection{Determination of Ion Densities}

[7] Random and systematic uncertainties in the INMS ion data are well understood. Measurement precision is limited by counting statistics and, in a typical scan, densities greater than $1 \mathrm{~cm}^{-3}$ are determined with a signal-to-noise ratio (SNR) of better than 5. In general, the dominant ions are measured with extremely good SNR throughout the altitude range of interest here. Densities are determined from measured count rates using prelaunch sensitivities measured in the laboratory [Waite et al., 2004; Cui et al., 2009]. These are estimated to be accurate to better than $25 \%$. However there are two potential complications, as addressed below.

[8] First, the angular acceptance of the INMS in the OSI mode is quite narrow with a half width at half maximum (HWHM) of $3^{\circ}$ for $\mathrm{M} / \mathrm{Z}=28$, when transmission is optimized at zero angle of attack [Waite et al., 2004]. Thus large tangential ion flows can shift the ambient ions away from optimal sensitivity. For a typical spacecraft velocity of $6 \mathrm{~km} \mathrm{~s}^{-1}$, a tangential flow speed of $0.5 \mathrm{~km} \mathrm{~s}^{-1}$ causes a decrease in sensitivity by $50 \%$ [Waite et al., 2004; Cravens et al., 2006]. Because the direction and magnitude of the ion flow are unknown, we do not attempt to derive the ion densities more accurately by correcting for possible ion drift motion. However, it should be born in mind that the ion densities given in this paper may not be reliable above $\sim 1450 \mathrm{~km}$, where transport starts to become significant and large ion flow velocities may occur [e.g., Ma et al., 2006; Cravens et al., 2008b].

[9] Second, the INMS transmission is also affected by spacecraft potential. We assume in our analysis a constant spacecraft potential of $-0.5 \mathrm{~V}$. An alternative scheme is to adopt the spacecraft potentials derived from other instruments (e.g., RPWS LP or CAPS IBS). Here we compare the ion densities obtained with the default value of $-0.5 \mathrm{~V}$ and those obtained with the RPWS spacecraft potential, to see how the derived densities could be affected by the choice of the spacecraft potential. A typical example is illustrated in Figure 1, for the outbound T26 flyby. In the left, we show the density profiles of three example ion species as a function of altitude, with the solid circles representing the densities calculated with the default spacecraft potential of $-0.5 \mathrm{~V}$ and the open circles representing the densities calculated with the RPWS spacecraft potential. These two choices of the spacecraft potential are also indicated in the right of Figure 1. The identification of the ion species shown in Figure 1 follows the scheme of Vuitton et al. [2007], with the numbers in parenthesis giving their $M / Z$ values. Figure 1 shows that typically, the uncertainties in ion densities associated with spacecraft potential are small, except at high altitudes where the spacecraft potential measured by RPWS deviates significantly from our default value of $-0.5 \mathrm{~V}$. It should be born in mind that adopting the RPWS spacecraft potential does not necessarily give more realistic results of the ambient ion densities compared with the default choice of $-0.5 \mathrm{~V}$, since the spacecraft potentials at different positions of the Cassini spacecraft may also be different. The above comparison is nevertheless instructive and suggests that the ion densities obtained at relatively low altitudes, typically below $\sim 1300 \mathrm{~km}$, are less dependent on 


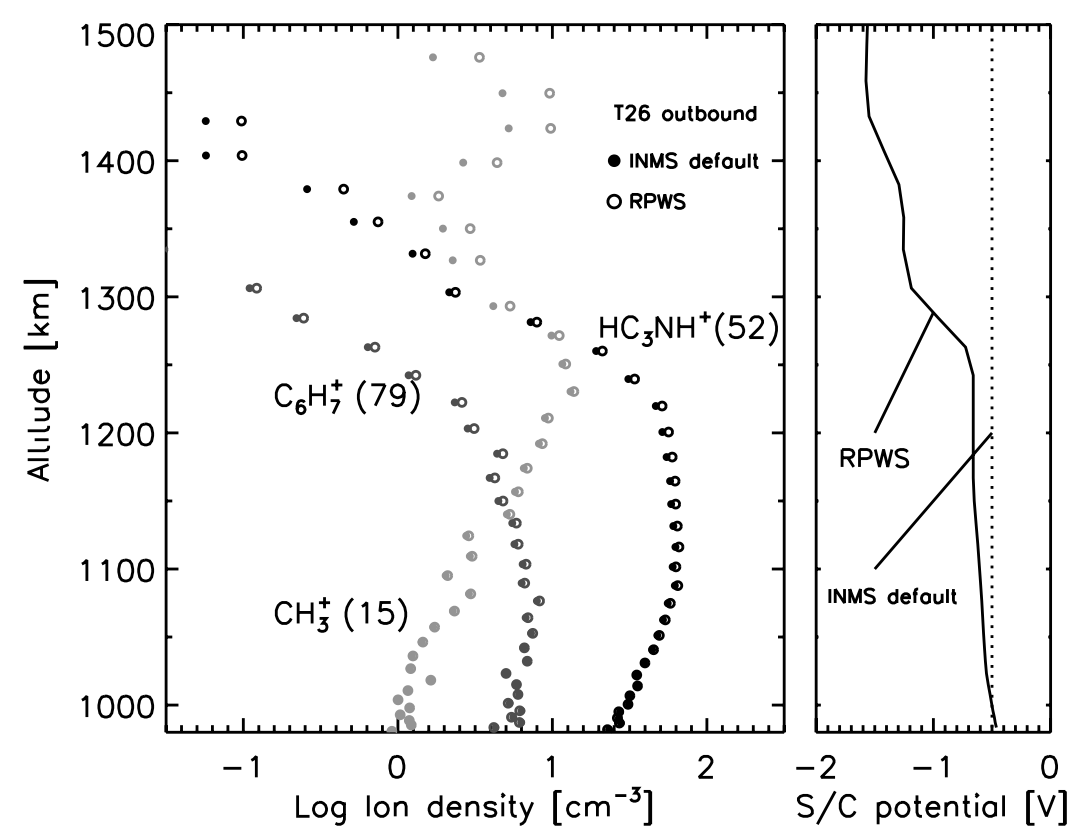

Figure 1. Comparison between the ion densities derived from different choices of the spacecraft potential for the outbound T26 flyby. The left gives the density profiles of three example ion species as a function of altitude. The identification of these ion species follows the scheme of Vuitton et al. [2007], with the numbers in parenthesis giving their $M / Z$ values. The solid circles represent the densities calculated with a constant spacecraft potential of $-0.5 \mathrm{~V}$ (the default INMS value), and the open circles represent those calculated with the RPWS spacecraft potential. The right indicates the two cases of the spacecraft potential used for calculating the ion densities.

spacecraft potential compared with those above. As the main results on the observed diurnal variations of Titan's ionosphere presented in this paper primarily rely on the measured ion densities below $\sim 1300 \mathrm{~km}$, both the adopted spacecraft potential and possible ion drift are not very likely to influence the main conclusions of this paper in any appreciable way.

[10] Similar to the INMS neutral data [Cui et al., 2009], in a typical ion spectrum there appears to be a broadly distributed background spectrum between $M / Z=10$ and 35, especially at low altitudes. We subtract these background signals with an empirical model to improve the density determination of the ambient ions. The adopted empirical background model is analogous to the one used for our previous analysis of the INMS neutral data [Cui et al., 2009]. For a given $M / Z$, the magnitude of background signals is found to depend exponentially on altitude, but show little variation between Titan's dayside and nightside. There also appears to be a minor bump at $M / Z<10$ for a typical ion spectrum, which cannot be accommodated with the empirical background model proposed above. Since the amplitude and shape of this minor bump of background counts are hard to constrain from the ion data, we exclude measurements in channels $1-10$ in our analysis, and as a consequence, we will not present in this study the ion abundances of species lighter than $\mathrm{C}^{+}(M / Z=12)$.

\subsection{Sample Selection}

[11] For the INMS ion data, different channels are not equally sampled, in the sense that signals in channels 16-17 and 28-29 are typically sampled with a frequency of $2 \mathrm{~Hz}$, whereas for the other channels, the frequency can be as low as $\sim 0.1 \mathrm{~Hz}$. Channels $16-17$ and $28-29$ are mainly associated with $\mathrm{CH}_{4}^{+}, \mathrm{CH}_{5}^{+}, \mathrm{HCNH}^{+}$and $\mathrm{C}_{2} \mathrm{H}_{5}^{+}$, with a small contribution from $\mathrm{NH}_{2}^{+}, \mathrm{NH}_{3}^{+}, \mathrm{C}_{2} \mathrm{H}_{4}^{+}$and $\mathrm{N}_{2} \mathrm{H}^{+}$[Vuitton et al., 2007]. The uneven sampling of different channels indicates that interpolation is required in order to extract an individual ion spectrum at a given altitude from the raw data. Here a common grid of time from closest approach (C/A) defined by channel 40 (mainly associated with $\mathrm{HC}_{2} \mathrm{NH}^{+}$) is chosen as the standard for interpolation. With this treatment, the sampling rates for channels $16-17$ and 28-29 are degraded.

[12] We include in this work the INMS ion data from 8 flybys, known in project parlance as T5, T17, T26, T32, T36, T37, T39 and T40. The T21 flyby occurred on Titan's nightside, and has been included in the recent work of Cravens et al. [2008b]. However, we exclude the T21 data from our sample since the corresponding ion density profiles show an abrupt transition near $1120 \mathrm{~km}$ by more than an order of magnitude, whereas the same feature is not observed in the associated RPWS electron density profile, known within 10\% [Ågren et al., 2009]. The RPWS spacecraft potential also presents a sharp transition at the same altitude. Considering these, we expect that the ion density profiles obtained from the T21 flyby are not reliable, and the sharp transition near $1120 \mathrm{~km}$ is more likely to be associated with instrumental effects rather than realistic features. The INMS ion data from the inbound T32 flyby are also excluded for the same reason. Ion densities on Titan's dayside could also be derived from the T18 data. However, during this flyby, the RPWS spacecraft potentials are significantly different from our default choice of $-0.5 \mathrm{~V}$, 
Table 1. Summary of the Trajectory Geometry at C/A for all Titan Flybys Used in This Study ${ }^{\mathrm{a}}$

\begin{tabular}{lccccccc}
\hline Flyby & Date & $\begin{array}{c}\text { ALT } \\
(\mathrm{km})\end{array}$ & $\begin{array}{c}\text { LST } \\
(\mathrm{hr})\end{array}$ & $\begin{array}{c}\text { SZA } \\
(\text { degree })\end{array}$ & $\begin{array}{c}\text { LAT } \\
(\text { degree })\end{array}$ & $\begin{array}{c}\text { LON } \\
(\text { degree })\end{array}$ & F10.7 \\
\hline T5 & 16 Apr 2005 & 1027 & 23.23 & 127 & 74 & 271 & 83 \\
T17 & 7 Sep 2006 & 1000 & 10.48 & 44 & 23 & 57 & 87 \\
T26 & 10 Mar 2007 & 981 & 1.78 & 149 & 32 & 358 & 71 \\
T32 & 13 Jun 2007 & 965 & 1.28 & 107 & 84 & 2 & 71 \\
T36 & 2 Oct 2007 & 973 & 16.14 & 67 & -60 & 109 & 66 \\
T37 & 19 Nov 2007 & 999 & 15.48 & 51 & -21 & 117 & 70 \\
T39 & 20 Dec 2007 & 970 & 11.50 & 61 & -70 & 177 & 73 \\
T40 & 5 Jan 2008 & 1014 & 14.54 & 38 & -12 & 130 & 80 \\
\hline
\end{tabular}

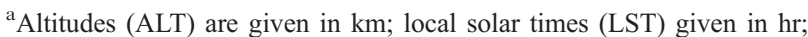
solar zenith angles (SZA), latitudes (LAT), and longitudes (LON) given in degrees; F10.7 solar fluxes (at $1 \mathrm{AU}$ ) given in units of $\mathrm{W} \mathrm{Hz}^{-1} \mathrm{~m}^{-2}$ multiplied by $10^{22}$.

by roughly a factor of $3-4$. Therefore to be conservative, the INMS ion data from T18 are also excluded.

[13] The main characteristics of all flybys in our sample at $\mathrm{C} / \mathrm{A}$ are detailed in Table 1, including the date of observation, altitude, local solar time, solar zenith angle, latitude, longitude and F10.7 solar flux at 1 AU (i.e., the $10.7 \mathrm{~cm}$ solar radio flux at $1 \mathrm{AU}$ in $\mathrm{W} \mathrm{Hz} \mathrm{m}^{-2}$ multiplied by $\left.10^{22}\right)$. Latitude is defined as northward positive. The longitude definition follows the scheme in which $0^{\circ}$ points to Saturn, $270^{\circ}$ points to the ideal magnetospheric ram direction, and $90^{\circ}$ points to the ideal magnetospheric wake direction. The data sampling covers more than two and a half years, between 16 April 2005 for T5 and 5 January 2008 for T40. Table 1 shows that altitudes at C/A range from $965 \mathrm{~km}$ for T32 to $1027 \mathrm{~km}$ for T5. The sample covers both Titan's northern and southern hemispheres, both equatorial and polar regions, as well as both the ideal magnetospheric ramside and wakeside. The F10.7 solar fluxes (at the Earth) are adopted from the daily values reported by the Dominion Radio Astrophysical Observatory at Penticton, B.C., Canada. The F10.7 value remains fairly constant from flyby to flyby, with a mean value of 75 and a standard deviation of less than $10 \%$. Thus we do not consider the variations of solar ionizing flux in our analysis. A more detailed description of the trajectory information for some of the flybys adopted in this work (including T5, T26, T32, T36 and T37) has been presented by Müller-Wodarg et al. [2008] and Cui et al. [2009]. However, the longitude definition used for this study is different from that by Müller-Wodarg et al. [2008].

[14] In the following sections, we will investigate the ion distributions for different solar zenith angle (hereafter SZA) bins. We will show that the observed ion spectra are strongly correlated with SZA. On physical grounds, variations with SZA and local solar time should encode similar physical information, but we choose SZA here since it is more directly related to solar ionization rates. Also, the data sampling with respect to local solar time is limited, especially near the morning terminator (see section 3 for details). We show in Figure 2 the sample distribution with respect to SZA, with different colors representing subsamples from different flybys. The number of ion spectra given in Figure 2 characterizes the spatial resolution of the sampling in mass channel 40, which is treated as a standard for interpolation to extract an individual ion spectrum (see section 2.1). Figure 2 shows that the INMS OSI measurements adopted in this study are mainly concentrated in three subgroups, the first one between $30^{\circ}$ and $70^{\circ}$, the second one between $70^{\circ}$ and $110^{\circ}$ and the third one beyond $120^{\circ}$. These roughly represent three distinct regimes from the sunlit side to the broad near-terminator transition region and then to the dark side of Titan [see also Ågren et al., 2009]. More specifically, Titan's dayside ionosphere is sampled by the INMS during the entire T17 and T40 flybys, the outbound T36 and T39 flybys, as well as part of the inbound T37 flyby below $1200 \mathrm{~km}$. The nightside is sampled during the entire T26 flyby and the outbound T5 flyby. The remaining part of the sample covers the near-terminator region of Titan's ionosphere, including the inbound T36 flyby (evening terminator), the outbound T32 flyby (morning terminator) as well as part of the inbound T37 above $\sim 1200 \mathrm{~km}$ (evening terminator).

[15] As pointed out by Müller-Wodarg et al. [2008] and Cui et al. [2009], one systematic uncertainty in analyzing the INMS data is because of the fact that the geometric information along the spacecraft trajectory may vary significantly, and it is oversimplistic to interpret the density profile of any species as a vertical distribution. This is especially important for this study, since the density profile of any ion species, when extracted along the spacecraft trajectory, is not illuminated by the Sun at fixed SZA. For instance, the SZA variation for the T40 flyby is as large as $\sim 51^{\circ}$ below $1500 \mathrm{~km}$. This fact brings some complexities in the interpretation and modeling of the observed ion distribution on Titan. To avoid these uncertainties, we combine all individual measurements, and for any given SZA and altitude bin, we determine the densities of different ion species by taking averages over all data points within that bin based on such a combined data set. We adopt throughout our analysis a fixed bin size of $50 \mathrm{~km}$ in altitude. The choice for different SZA bins is based on the sample distribution shown in Figure 2, i.e., one dayside bin at

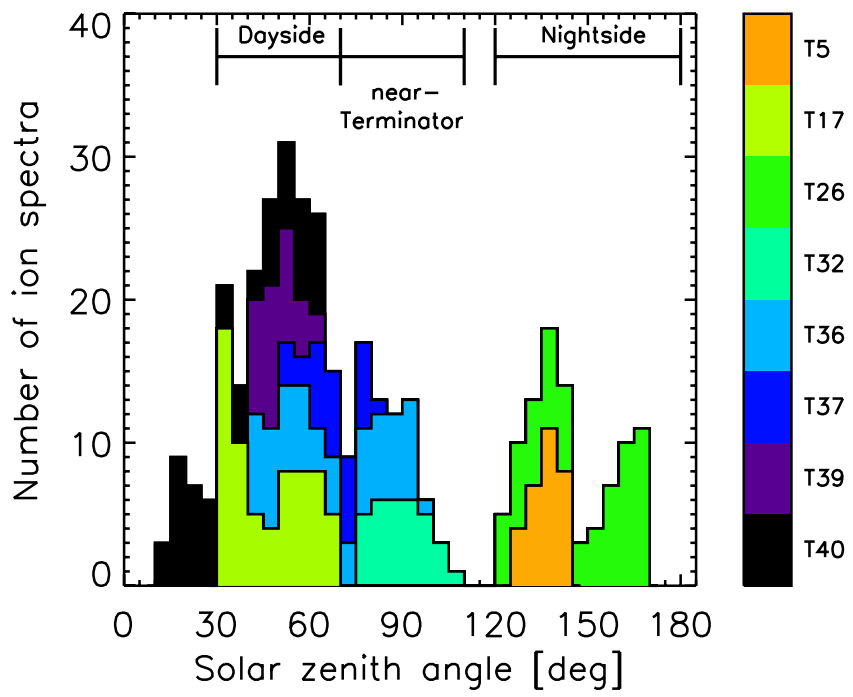

Figure 2. Distribution of solar zenith angle (SZA) for the sample adopted in this study. Different colors represent subsamples from different flybys. The SZA distribution is characterized by three distinct groups, one between $30^{\circ}$ and $70^{\circ}$ (dayside), one between $70^{\circ}$ and $110^{\circ}$ (near terminator), and the other one beyond $120^{\circ}$ (nightside). 

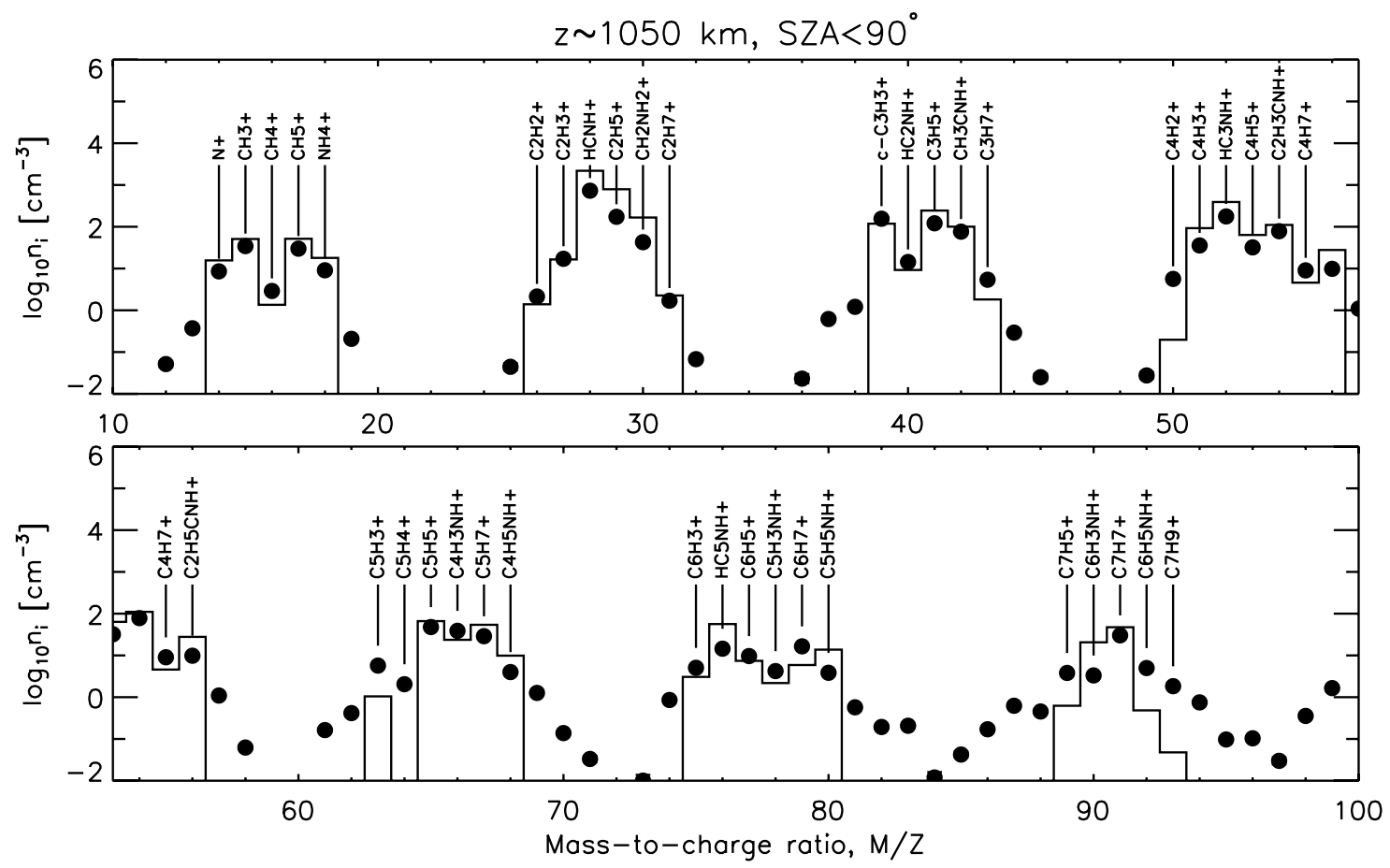

Figure 3. Observed ion mass spectrum averaged between 1025 and $1075 \mathrm{~km}$ and averaged over Titan's dayside (with SZA smaller $90^{\circ}$ ). The spectrum is characterized by seven distinct groups, with a mean mass spacing of $\sim 12 \mathrm{Da}$. The solid line shows the dayside ion spectrum, calculated with the timedependent ion chemistry model described in section 5. The model spectrum is shown for a local solar time of $9.5 \mathrm{hr}$, roughly consistent with the mean SZA value on Titan's dayside for our sample. The ion species that account for the bulk signals in those mass channels with densities greater than $1 \mathrm{~cm}^{-3}$ are labeled. The identification of these ion species follows the scheme of Vuitton et al. [2007].

$30^{\circ}-70^{\circ}$, one near-terminator bin at $70^{\circ}-110^{\circ}$, and one nightside bin beyond $120^{\circ}$. However, in some cases we will distinguish between the morning and evening terminators. We will show that the difference between the dawn and dusk ion density profiles has some interesting implications (see section 4 for details).

\section{Results}

[16] An example of the ion mass spectrum extracted from the combined INMS data is given in Figure 3, for an altitude of $\sim 1050 \mathrm{~km}$ with a bin size of $50 \mathrm{~km}$. The background spectrum has been subtracted, and all measurements obtained on Titan's dayside (with SZA smaller than $90^{\circ}$ ) are included and averaged. Some obvious features are immediately seen. First, there is no unique ion species that dominates Titan's ionosphere, in contrast to the neutral gas distribution for which $\mathrm{N}_{2}$ has a mixing ratio of over $95 \%$ [Waite et al., 2005; Yelle et al., 2006, 2008; Cui et al., 2008, 2009]. The most abundant ion species are $\mathrm{HCNH}^{+}(7.4 \times$ $\left.10^{2} \mathrm{~cm}^{-3}\right)$, followed by $\mathrm{C}_{2} \mathrm{H}_{5}^{+}\left(1.7 \times 10^{2} \mathrm{~cm}^{-3}\right), \mathrm{c}^{-} \mathrm{C}_{3} \mathrm{H}_{3}^{+}$ $\left(1.5 \times 10^{2} \mathrm{~cm}^{-3}\right), \mathrm{HC}_{3} \mathrm{NH}^{+}\left(1.7 \times 10^{2} \mathrm{~cm}^{-3}\right)$ and $\mathrm{C}_{3} \mathrm{H}_{5}^{+}$ $\left(1.2 \times 10^{2} \mathrm{~cm}^{-3}\right)$, where all density values refer to an altitude of $1050 \mathrm{~km}$ averaged over Titan's dayside. This is in general agreement with early chemical model predictions [e.g., Keller et al., 1992, 1998; Fox and Yelle, 1997]. The ion spectrum is characterized by seven distinct groups with mass ranges of $10-25,25-35,35-45,45-60,60-70,70-$ 85 , and beyond 85 , respectively. The mean mass spacing between these groups is $\sim 12 \mathrm{Da}$, based on a linear fitting of the mean ion mass of each group [see also Cravens et al., 2006; Vuitton et al., 2007]. In most cases, the signals in a given channel are primarily contributed by a single ion species, with only a small fraction (typically less than $10 \%$ ) from other contaminating minor ions as inferred from photochemical model calculations [e.g., Vuitton et al., 2007]. The primary ion species that account for the bulk signals in channels with significant count rates, or with number densities greater than $1 \mathrm{~cm}^{-3}$ at $\sim 1050 \mathrm{~km}$, are labeled in Figure 3 for reference. The solid line in Figure 3 gives the dayside ion spectrum at $9.5 \mathrm{hr}$ local solar time, calculated with the time-dependent ion chemistry model described in section 5 .

[17] We show in Figure 4 a direct comparison between the ion mass spectra on Titan's dayside (open circles) and nightside (solid circles), respectively. These spectra are obtained by averaging over the combined INMS data set with SZA in the range of $30^{\circ}-70^{\circ}$ (dayside) or $120^{\circ}-180^{\circ}$ (nightside), both centered at an altitude of $1050 \mathrm{~km}$ with a bin size of $50 \mathrm{~km}$. The background signals have been subtracted from the raw spectra (see section 2.1). Figure 4 shows that the densities of most ion species vary significantly with SZA, with the nightside presenting an overall ion depletion. This is consistent with the diurnal variations of electron distribution revealed by the RPWS LP data [Agren et al., 2009], and confirms the importance of solar radiation as the main ion source for Titan's dayside ionosphere, though the satellite is subject to a solar radiation flux 

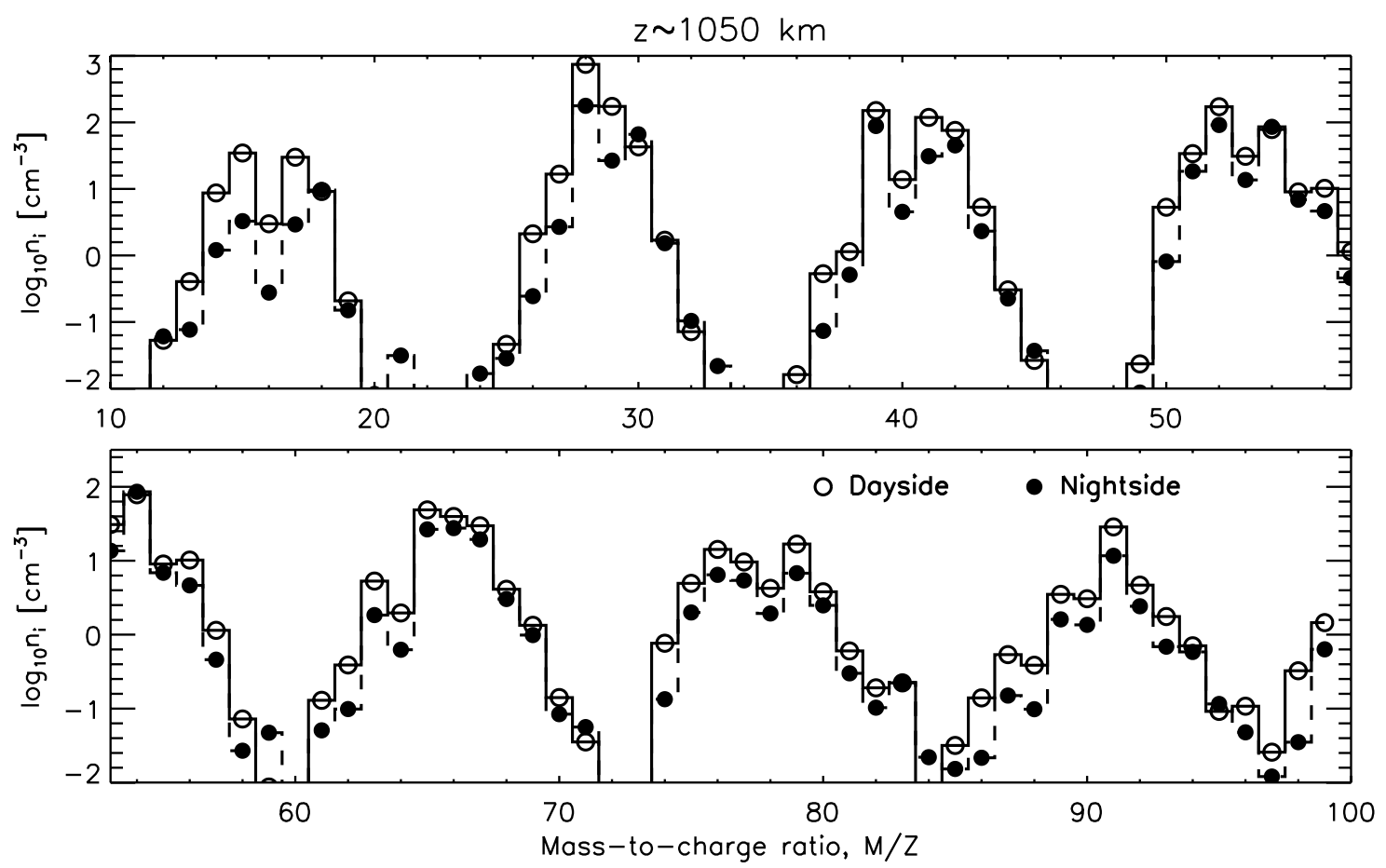

Figure 4. Observed ion mass spectra averaged between 1025 and $1075 \mathrm{~km}$, with the dayside spectrum (SZA between $30^{\circ}$ and $70^{\circ}$ ) and nightside spectrum (SZA beyond $120^{\circ}$ ) displayed with open and solid circles, respectively. Figure 4 shows clear diurnal variation of Titan's ionosphere, with a general trend of ion depletion on the nightside. However, such a diurnal variation is more prominent for light ions than for heavy ones.

$\sim 100$ times weaker than that at Earth and is, for most of the time, subject to precipitating particles from Saturn's magnetosphere.

[18] We show in Figure 5 the total ion density profiles for different conditions of solar illumination. The INMS ion distributions near the morning and evening terminators are presented separately. However, the profile for the morning terminator (the dashed line in Figure 5) cannot be regarded as vertical since it comes from a single flyby (outbound T32) and the associated variation in SZA is quite large along the spacecraft trajectory. The total ion densities shown in Figure 5 are obtained by summing all ion species over the INMS mass range up to $M / Z=99$. We ignore throughout this paper the heavy positive ions with $M / Z>100$ since they cannot be detected by the INMS [e.g., Waite et al., 2007; F. Crary et al., Heavy and negative ions at Titan measured by Cassini CAPS, paper presented at 37th COSPAR Scientific Assembly, Montreal, Canada, 13-20 Jul., 2008; J.-E. Wahlund et al., On the amount of heavy molecular ions in Titan's ionosphere, submitted to Planetary and Space Science, 2009, hereinafter referred to as Wahlund et al., submitted manuscript, 2009]. Though these ions may contribute to a nonnegligible fraction of the total ion content (e.g., Wahlund et al., submitted manuscript, 2009), the main conclusions in this paper, which are based on the observations of individual ion species, are not affected by such a biased sampling.

[19] The INMS ion distribution on Titan's dayside shows a well-defined ionization peak at $\sim 1120 \mathrm{~km}$, with a peak density of $\sim 2.4 \times 10^{3} \mathrm{~cm}^{-3}$ (averaged over the SZA range of $30^{\circ}-70^{\circ}$ ). A similar structure is observed near both the morning and evening terminators, as a result of the large extension of Titan's atmosphere [Müller-Wodarg et al., 2000; Cravens et al., 2004; Agren et al., 2009]. The INMS

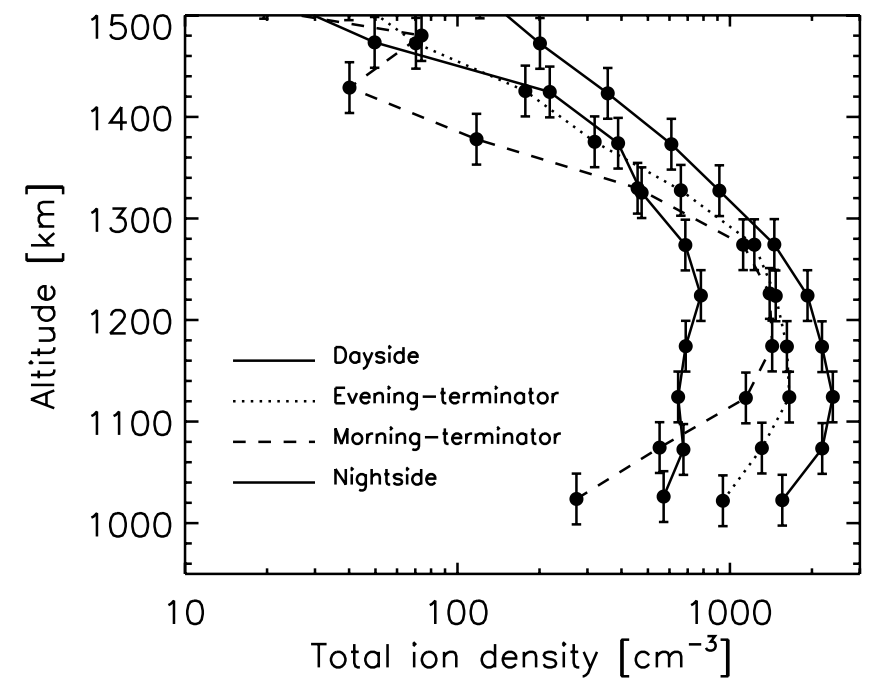

Figure 5. Observed total ion density profiles in Titan's ionosphere for different conditions of solar illumination. The dayside ion distribution shows a well-defined ionization peak. A similar feature is observed near both the morning and evening terminators. There is also clear asymmetry between the ion density profiles observed near the morning and evening terminators, with the ion densities at dawn significantly lower than at dusk. The nightside distribution becomes quite flat below $\sim 1300 \mathrm{~km}$. 


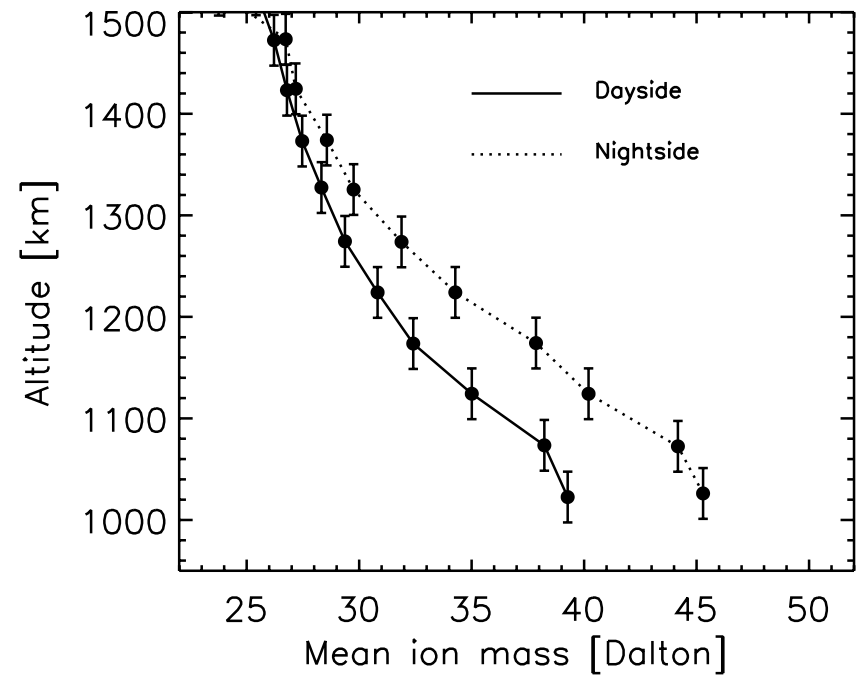

Figure 6. Observed altitude dependence of mean ion mass (weighted by number density) for the dayside (solid line) and nightside (dotted line) of Titan. The mean ion mass increases with decreasing altitude in all cases. The diurnal variation of Titan's ionospheric composition is most prominent at low altitudes.

ion distribution near the evening terminator peaks at roughly the same altitude as on the dayside, but with a reduced peak ion density of $\sim 1.7 \times 10^{3} \mathrm{~cm}^{-3}$ (from the inbound T36 and part of the inbound T37 data). The ionization peak at the morning terminator shifts upward to $\sim 1170 \mathrm{~km}$, with a peak ion density of $\sim 1.4 \times 10^{3} \mathrm{~cm}^{-3}$ (from the outbound T32 data). There is a clear asymmetry between the ion density profiles observed near the morning and evening terminators, in that the ion densities at the morning terminator are significantly lower than those at the evening terminator. Finally, the INMS ion distribution observed far beyond the terminator (with $\mathrm{SZA}>120^{\circ}$ ) becomes quite flat below $\sim 1300 \mathrm{~km}$, characterized by a density plateau of $\sim 700 \mathrm{~cm}^{-3}$. There is no clear evidence for any existing ionization peak on the nightside. Similar plateaus are also observed in the nightside electron density profiles measured by RPWS [Ägren et al., 2009].

[20] On the basis of the ion spectra shown in Figure 4, it appears that the diurnal variation is most prominent for light ions, and becomes less pronounced for heavy ones. Specifically, the ion content on Titan's nightside compared with the dayside is on average depleted by a factor of $\sim 5$ for the mass group of $M / Z=15-25$, and depleted by a factor of $\sim 4$ for the mass group of $M / Z=25-35$. For all heavier groups, the ion densities are depleted on the nightside compared with the dayside by roughly a factor of $\sim 2$.

[21] The ionospheric composition can be characterized by the parameter of mean ion mass, weighted by number density. Figure 6 shows the altitude dependence of the calculated mean ion mass for the dayside and nightside of Titan, respectively. Note that the profiles of mean ion mass shown in Figure 6 are biased because of the presence of heavy positive ions with $M / Z>100$ not sampled by the INMS, especially at low altitudes [Waite et al., 2007; Crary et al., presented paper, 2008; Wahlund et al., submitted manuscript, 2009]. Despite this, Figure 6 still provides a

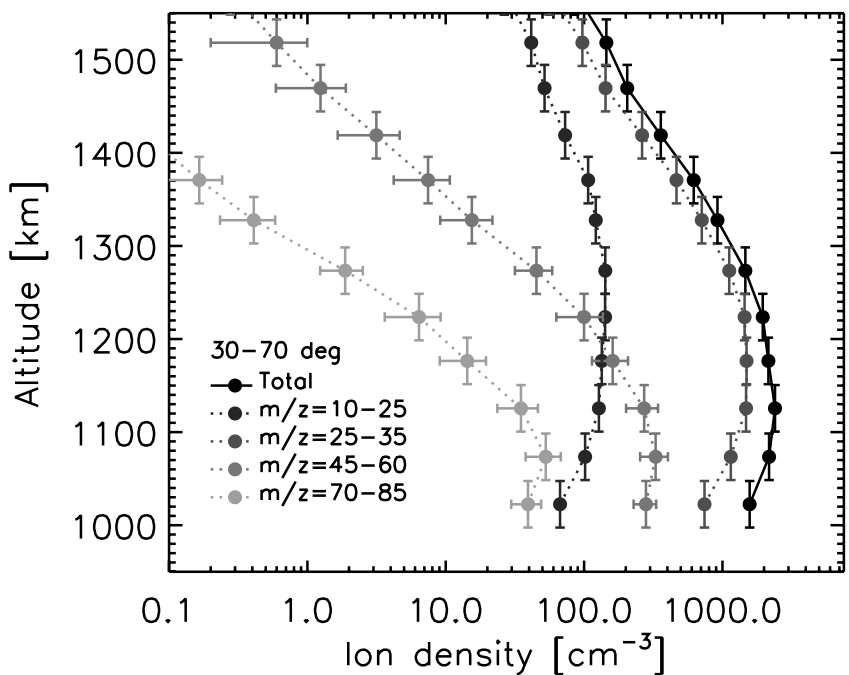

Figure 7. Observed density profiles of different ion groups (dotted lines) and the total ion density profile (solid line) for Titan's dayside ionosphere.

qualitatively interesting picture for the diurnal variation of Titan's ionospheric composition. On both the dayside and nightside, the mean ion mass increases with decreasing altitude, implying a larger contribution from heavy ions at low altitudes. Figure 6 also reveals that the nightside of Titan presents a larger contribution from heavy ions compared with the dayside. More specifically, at $\sim 1050 \mathrm{~km}$, the contribution of all ions with $M / Z>45$ is $\sim 27 \%$ of the total ion density on the dayside, but increases to $\sim 43 \%$ on the nightside. Such a difference is less obvious at high altitudes, where the ionospheric composition is dominated by light ions for both the dayside and nightside.

[22] To investigate further the diurnal variations of different ion species, we show in Figure 7 (for the dayside) and Figure 8 (for the nightside) the density profiles of various ion groups, as defined by the apparent mass peaks observed in Figures 3 and 4. Figures 7 and 8 suggest that on both the

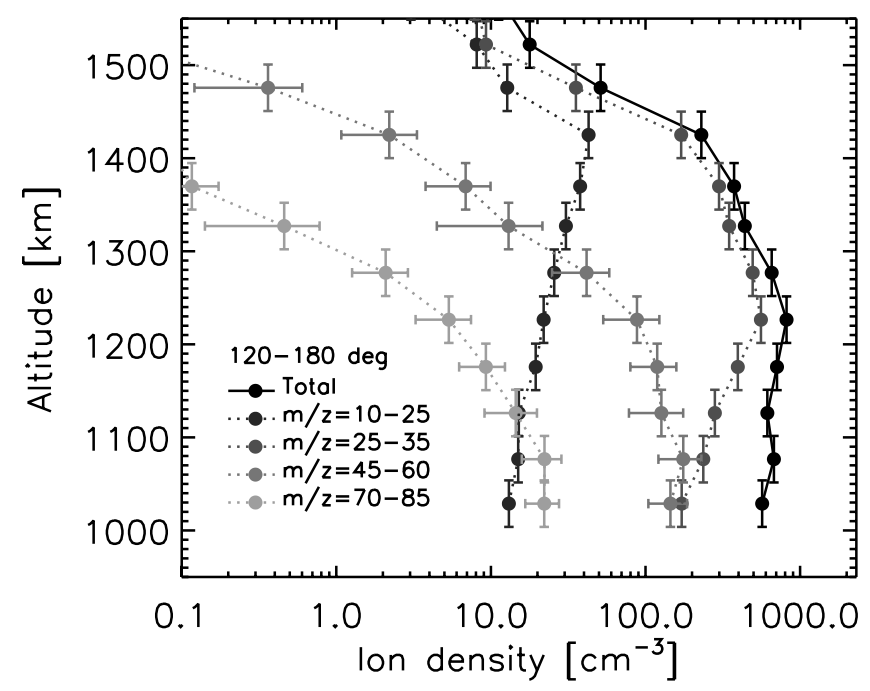

Figure 8. Observed density profiles of different ion groups (dotted lines) and the total ion density profile (solid line) for Titan's nightside ionosphere. 


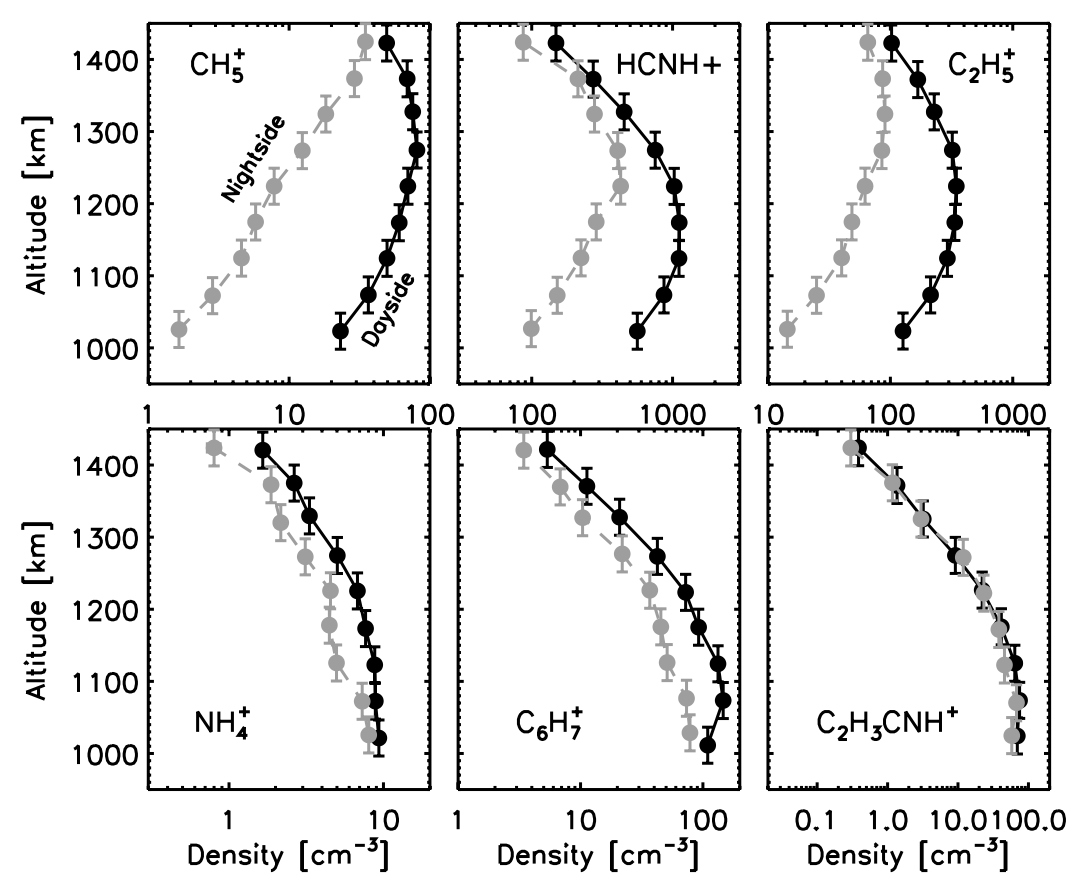

Figure 9. Observed diurnal variations of several representative ion species. Black and gray circles represent the ion density profiles on Titan's dayside and nightside, respectively. All species in the top show clear nightside depletion at low altitudes, whereas those in the bottom present modest nightside depletion at all altitudes.

dayside and nightside of Titan, the density profiles of light species show well-defined ionization peaks. In contrast, the observed heavy ion profiles do not show clear signatures of ionization peak between 1000 and $1300 \mathrm{~km}$, especially on the nightside. Titan's dayside ionosphere is dominated by relatively light ions, especially the mass group of $M / Z=$ 25-35. As a consequence, the dayside total ion density profile is also characterized by a well-defined ionization peak, as shown by the solid line in Figure 5. At low altitudes, the densities of the light ion species decrease significantly from the dayside to the nightside, shifting the density peak toward higher altitudes. In contrast, the heavy ions show modest diurnal variations and make a significant contribution to the total ion density on the nightside. The combined result is the nightside plateau below $\sim 1300 \mathrm{~km}$ observed in the total ion density profile (and RPWS electron distribution [Ägren et al., 2009]), as revealed by the dashdotted line in Figure 5.

[23] The extent of ion depletion on Titan's nightside is more clearly seen in Figure 9, where we present the density profiles of several representative ion species, including $\mathrm{CH}_{5}^{+}, \mathrm{HCNH}^{+}, \mathrm{C}_{2} \mathrm{H}_{5}^{+}, \mathrm{NH}_{4}^{+}, \mathrm{C}_{6} \mathrm{H}_{7}^{+}$and $\mathrm{C}_{2} \mathrm{H}_{3} \mathrm{CNH}^{+}$. The black and gray circles represent the profiles averaged on Titan's dayside and nightside, respectively. On one hand, the nightside profiles of the three species in the top show clear density decrement at low altitudes. On the other hand, such a feature is not observed for the three species shown in the bottom of Figure 9, which only present modest depletion at all altitudes. It is also interesting to note that except for $\mathrm{NH}_{4}^{+}$, all species in the top are lighter than those in the bottom. Therefore although there is a general trend that light ions typically show clear density decrement on the nightside (thus more prominent diurnal variations), whereas the nightside depletion of heavy ions is less pronounced, there are indeed exceptions, such as $\mathrm{NH}_{4}^{+}$, which belong to light species but present day-to-night differences characteristic of typical heavy ions.

[24] We summarize in Table 2 the observed ion densities averaged on Titan's dayside (with SZA between $30^{\circ}$ and $70^{\circ}$ ) and nightside (with SZA beyond $120^{\circ}$ ) at $\sim 1050 \mathrm{~km}$, as well as the corresponding night-to-day density ratios. Only species with dayside densities greater than $1 \mathrm{~cm}^{-3}$ are listed. The lifetimes (or loss time constants) of these ions averaged over a Titan day and at an altitude of $1050 \mathrm{~km}$ are also given in Table 2. The ionosphere model used to calculate these ion lifetimes is described in section 5. In Figure 10, we present the relation between the observed night-to-day density ratio and the calculated ion lifetime, for all species identified in the INMS ion spectra with dayside density at $1050 \mathrm{~km}$ greater than $0.1 \mathrm{~cm}^{-3}$. Figure 10 shows a clear correlation between the ion lifetime and the observed night-to-day ratio, demonstrating that the loss time constant is an important factor in interpreting the diurnal variations in ionospheric densities.

[25] The scattering of the data points in Figure 10 is nevertheless relatively large, especially for long-lived ions with lifetimes greater than $100 \mathrm{sec}$. There are at least two possible interpretations for this. First, the derived lifetimes for some ions may not be accurate, since the reaction rates, especially for the heavy ions, are poorly constrained [Carrasco et al., 2008]. For ions with no existing lab measurement of their reaction rates, we assume their recombination coefficients to be $\alpha(T)=7 \times$ $10^{-7}(T / 300)^{-0.7} \mathrm{~cm}^{-3} \mathrm{~s}^{-1}$. However, recent flowing afterglow studies of the dissociative recombination of several hydrocarbon ions suggest instead a switch in power index from $\sim-0.7$ to $\sim-1.4$ for electron temperatures higher than $300 \mathrm{~K}$ [McLain et al., 2004]. This implies that the 
Table 2. Globally Averaged Ion Densities Observed on Titan's Dayside and Nightside at an Altitude of $1050 \mathrm{~km}^{\mathrm{a}}$

\begin{tabular}{|c|c|c|c|c|c|}
\hline Species & $M / Z$ & $N_{i}^{\text {(day) }}\left(\mathrm{cm}^{-3}\right)$ & $N_{i}^{\text {(night) }}\left(\mathrm{cm}^{-3}\right)$ & $\frac{N_{i}^{\text {(night) }}}{N_{i}^{\text {(day) }}}$ & $t_{\text {chem }}(\mathrm{s})$ \\
\hline $\mathrm{N}^{+}$ & 14 & 8.7 & 1.2 & 0.14 & 6.5 \\
\hline $\mathrm{CH}_{3}^{+}$ & 15 & $3.5(+1)$ & 3.3 & 0.095 & 7.1 \\
\hline $\mathrm{CH}_{4}^{+}$ & 16 & 3.0 & $2.8(-1)$ & 0.093 & 6.4 \\
\hline $\mathrm{CH}_{5}^{+}$ & 17 & $3.0(+1)$ & 2.9 & 0.098 & $5.6(+1)$ \\
\hline $\mathrm{NH}_{4}^{+}$ & 18 & 9.1 & 9.6 & 1.05 & $3.2(+2)$ \\
\hline $\mathrm{C}_{2} \mathrm{H}_{2}^{+}$ & 26 & 2.1 & $2.4(-1)$ & 0.12 & 8.7 \\
\hline $\mathrm{C}_{2} \mathrm{H}_{3}^{+}$ & 27 & $1.7(+1)$ & 2.7 & 0.16 & $3.2(+1)$ \\
\hline $\mathrm{HCNH}^{+}$ & 28 & $7.4(+2)$ & $1.8(+2)$ & 0.24 & $5.5(+2)$ \\
\hline $\mathrm{C}_{2} \mathrm{H}_{5}^{+}$ & 29 & $1.7(+2)$ & $2.7(+1)$ & 0.15 & $1.1(+2)$ \\
\hline $\mathrm{CH}_{2} \mathrm{NH}_{2}^{+}$ & 30 & $4.3(+1)$ & $6.6(+1)$ & 1.54 & $1.6(+3)$ \\
\hline $\mathrm{C}_{2} \mathrm{H}_{7}^{+}$ & 31 & 1.7 & 1.5 & 0.91 & $1.7(+2)$ \\
\hline $\mathrm{CNC}^{+}$ & 38 & 1.1 & $5.1(-1)$ & 0.45 & $3.0(+2)$ \\
\hline $\mathrm{c}-\mathrm{C}_{3} \mathrm{H}_{3}^{+}$ & 39 & $1.5(+2)$ & $8.8(+1)$ & 0.58 & $5.8(+2)$ \\
\hline $\mathrm{HC}_{2} \mathrm{NH}^{+}$ & 40 & $1.4(+1)$ & 4.5 & 0.33 & $1.4(+3)$ \\
\hline $\mathrm{C}_{3} \mathrm{H}_{5}^{+}$ & 41 & $1.2(+2)$ & $3.1(+1)$ & 0.26 & $1.4(+2)$ \\
\hline $\mathrm{CH}_{3} \mathrm{CNH}^{+}$ & 42 & $7.6(+1)$ & $4.5(+1)$ & 0.60 & $1.4(+3)$ \\
\hline $\mathrm{C}_{3} \mathrm{H}_{7}^{+}$ & 43 & 5.3 & 2.3 & 0.44 & $1.8(+2)$ \\
\hline $\mathrm{C}_{4} \mathrm{H}_{2}^{+}$ & 50 & 5.3 & $8.1(-1)$ & 0.15 & $1.1(+2)$ \\
\hline $\mathrm{C}_{4} \mathrm{H}_{3}^{+}$ & 51 & $3.4(+1)$ & $1.8(+1)$ & 0.54 & $3.7(+2)$ \\
\hline $\mathrm{HC}_{3} \mathrm{NH}^{+}$ & 52 & $1.7(+2)$ & $9.1(+1)$ & 0.53 & $2.3(+2)$ \\
\hline $\mathrm{C}_{4} \mathrm{H}_{5}^{+}$ & 53 & $3.1(+1)$ & $1.4(+1)$ & 0.44 & $3.4(+2)$ \\
\hline $\mathrm{C}_{2} \mathrm{H}_{3} \mathrm{CNH}^{+}$ & 54 & $7.8(+1)$ & $8.6(+1)$ & 1.10 & $8.5(+2)$ \\
\hline $\mathrm{C}_{4} \mathrm{H}_{7}^{+}$ & 55 & 9.0 & 6.9 & 0.76 & $4.6(+2)$ \\
\hline $\mathrm{C}_{2} \mathrm{H}_{5} \mathrm{CNH}^{+}$ & 56 & $1.0(+1)$ & 4.6 & 0.46 & $9.6(+2)$ \\
\hline $\mathrm{C}_{4} \mathrm{H}_{9}^{+}$ & 57 & 1.1 & $4.6(-1)$ & 0.40 & $4.6(+2)$ \\
\hline $\mathrm{C}_{5} \mathrm{H}_{3}^{+}$ & 63 & 5.3 & 1.8 & 0.35 & $4.1(+2)$ \\
\hline $\mathrm{C}_{5} \mathrm{H}_{4}^{+}$ & 64 & 2.0 & $6.3(-1)$ & 0.32 & $4.7(+2)$ \\
\hline $\mathrm{C}_{5} \mathrm{H}_{5}^{+}$ & 65 & $4.9(+1)$ & $2.7(+1)$ & 0.54 & $3.8(+2)$ \\
\hline $\mathrm{C}_{4} \mathrm{H}_{3} \mathrm{NH}^{+}$ & 66 & $4.0(+1)$ & $2.8(+1)$ & 0.70 & $8.9(+2)$ \\
\hline $\mathrm{C}_{5} \mathrm{H}_{7}^{+}$ & 67 & $3.0(+1)$ & $1.9(+1)$ & 0.65 & $4.5(+2)$ \\
\hline $\mathrm{C}_{4} \mathrm{H}_{5} \mathrm{NH}^{+}$ & 68 & 4.1 & 3.0 & 0.74 & $9.4(+2)$ \\
\hline $\mathrm{C}_{5} \mathrm{H}_{9}^{+}$ & 69 & 1.3 & $9.9(-1)$ & 0.74 & $4.6(+2)$ \\
\hline $\mathrm{C}_{6} \mathrm{H}_{3}^{+}$ & 75 & 4.9 & 2.0 & 0.41 & $3.4(+2)$ \\
\hline $\mathrm{HC}_{5} \mathrm{NH}^{+}$ & 76 & $1.4(+1)$ & 6.4 & 0.45 & $8.9(+2)$ \\
\hline $\mathrm{C}_{6} \mathrm{H}_{5}^{+}$ & 77 & 9.6 & 5.4 & 0.56 & $7.6(+1)$ \\
\hline $\mathrm{C}_{5} \mathrm{H}_{3} \mathrm{NH}^{+}$ & 78 & 4.2 & 1.9 & 0.46 & $9.9(+2)$ \\
\hline $\mathrm{C}_{6} \mathrm{H}_{7}^{+}$ & 79 & $1.7(+1)$ & 6.8 & 0.40 & $2.8(+2)$ \\
\hline $\mathrm{C}_{5} \mathrm{H}_{5} \mathrm{NH}^{+}$ & 80 & 3.8 & 2.5 & 0.66 & $8.9(+2)$ \\
\hline $\mathrm{C}_{7} \mathrm{H}_{5}^{+}$ & 89 & 3.5 & 1.6 & 0.46 & $8.9(+2)$ \\
\hline $\mathrm{C}_{6} \mathrm{H}_{3} \mathrm{NH}^{+}$ & 90 & 3.1 & 1.4 & 0.44 & $8.9(+2)$ \\
\hline $\mathrm{C}_{7} \mathrm{H}_{7}^{+}$ & 91 & $2.9(+1)$ & $1.2(+1)$ & 0.41 & $6.1(+2)$ \\
\hline $\mathrm{C}_{6} \mathrm{H}_{5} \mathrm{NH}^{+}$ & 92 & 4.7 & 2.4 & 0.52 & $1.5(+3)$ \\
\hline $\mathrm{C}_{7} \mathrm{H}_{9}^{+}$ & 93 & 1.8 & $6.9(-1)$ & 0.39 & $5.3(+2)$ \\
\hline $\mathrm{C}_{8} \mathrm{H}_{3}^{+}$ & 99 & 1.5 & $6.3(-1)$ & 0.44 & $4.1(+2)$ \\
\hline
\end{tabular}

${ }^{a}$ Also given in the table are the observed night-to-day ion density ratios and the associated ion lifetimes. The ion lifetimes are estimated from the time-dependent ion chemistry model described in section 5 . Values with format $a(n)$ represent $a \times 10^{n}$.

actual dissociative recombination rates could be a factor of 2 lower than what we have adopted, and thus the ion lifetimes could be significantly underestimated. This however does not affect the estimation for light ions since their reaction rates are relatively well constrained from lab measurements. Second, the mass channels associated with some species are likely to be appreciably contaminated by other ions (or isomers). As an example, our ion chemistry model (see section 5 for details) shows that the observed diurnal variation of $\mathrm{HC}_{2} \mathrm{NH}^{+}$is likely to be appreciably contaminated by $\mathrm{C}_{3} \mathrm{H}_{4}^{+}$. Both species have $M / Z=40$ with the latter having a number fraction of $\sim 30 \%$ averaged over a Titan day, at an altitude of $1050 \mathrm{~km}$. The associated lifetimes of the two species are very different, estimated to be $1.4 \times 10^{3}$ sec for $\mathrm{HC}_{2} \mathrm{NH}^{+}$and $\sim 80 \mathrm{sec}$ for $\mathrm{C}_{3} \mathrm{H}_{4}^{+}$.
[26] On the basis of the findings presented above, the characteristics of the diurnal variations of Titan's ionosphere can be summarized as follows.

[27] 1. The total ion density distribution shows welldefined ionization peak on the dayside of Titan. On the nightside, though there is an overall ion depletion compared with the dayside, the total ion content is still appreciable. The nightside total ion density profile is characterized by a flat distribution at a level of $\sim 700 \mathrm{~cm}^{-3}$ below $\sim 1300 \mathrm{~km}$. Such a flat distribution is the combined result of the distinct nightside profiles of light and heavy ion populations.

[28] 2. There is a clear asymmetry between the ion density profiles observed near the morning and evening terminators, in that ion densities near the morning terminator are smaller than those near the evening terminator.

[29] 3. The observed diurnal variation is most prominent for light ions (e.g., $\mathrm{CH}_{5}^{+}, \mathrm{HCNH}^{+}, \mathrm{C}_{2} \mathrm{H}_{5}^{+}$). In contrast, only modest diurnal variation is observed for heavy ones (e.g., $\mathrm{C}_{2} \mathrm{H}_{3} \mathrm{CNH}^{+}, \mathrm{C}_{6} \mathrm{H}_{7}^{+}$).

[30] 4. Despite the abovementioned general trend, there are exceptions with some light ions, such as $\mathrm{NH}_{4}^{+}$, showing day-to-night differences characteristic of typical heavy ones.

[31] 5. The observed diurnal variations of different ion species, as quantified by the measured night-to-day ion density ratios, are strongly correlated with the associated chemical lifetimes. Possible interpretations of these observations are presented in section 4.

\section{Interpretation: Chemical Survival of Dayside Ions as an Important Source for Titan's Nightside Ionosphere}

[32] The primary characteristics of the diurnal variations of Titan's ionosphere are clearly a result of chemical loss of ions as Titan rotates into darkness and ion production initiated by solar illumination is turned off [Vuitton et al., 2007]. Several characteristics seen in the INMS data are consistent with maintenance of the nightside ionosphere by chemical survival of dayside ions.

[33] The scenario of chemical survival of dayside ions is consistent with the correlation between the observed nightto-day ion density ratios and the associated ion lifetimes, as shown in Figure 10. Such a scenario also naturally explains the distinctions between the diurnal variations of light and heavy ions, which are associated with their different chemical loss pathways. The light ions are primarily lost through ion-neutral chemistry whereas the heavy ions are primarily lost through electron dissociative recombination [Vuitton et al., 2007]. In Titan's upper atmosphere, the typical recombination time constant is significantly larger than the typical chemical time constant for ion-neutral reactions (see Table 2). This is primarily associated with the difference in density between electrons and neutrals [e.g., Vuitton et al., 2007]. As a consequence, when Titan rotates into darkness with photoionization turned off, light ions are not able to survive fast loss through ion-neutral chemistry, and thus are observed to be significantly depleted on the nightside. On the contrary, heavy ions may survive recombination because of their relatively slow loss. The observed diurnal variation of $\mathrm{NH}_{4}^{+}$as shown in Figure 9 is also consistent with such a scenario, since $\mathrm{NH}_{4}^{+}$is primarily lost through electron 


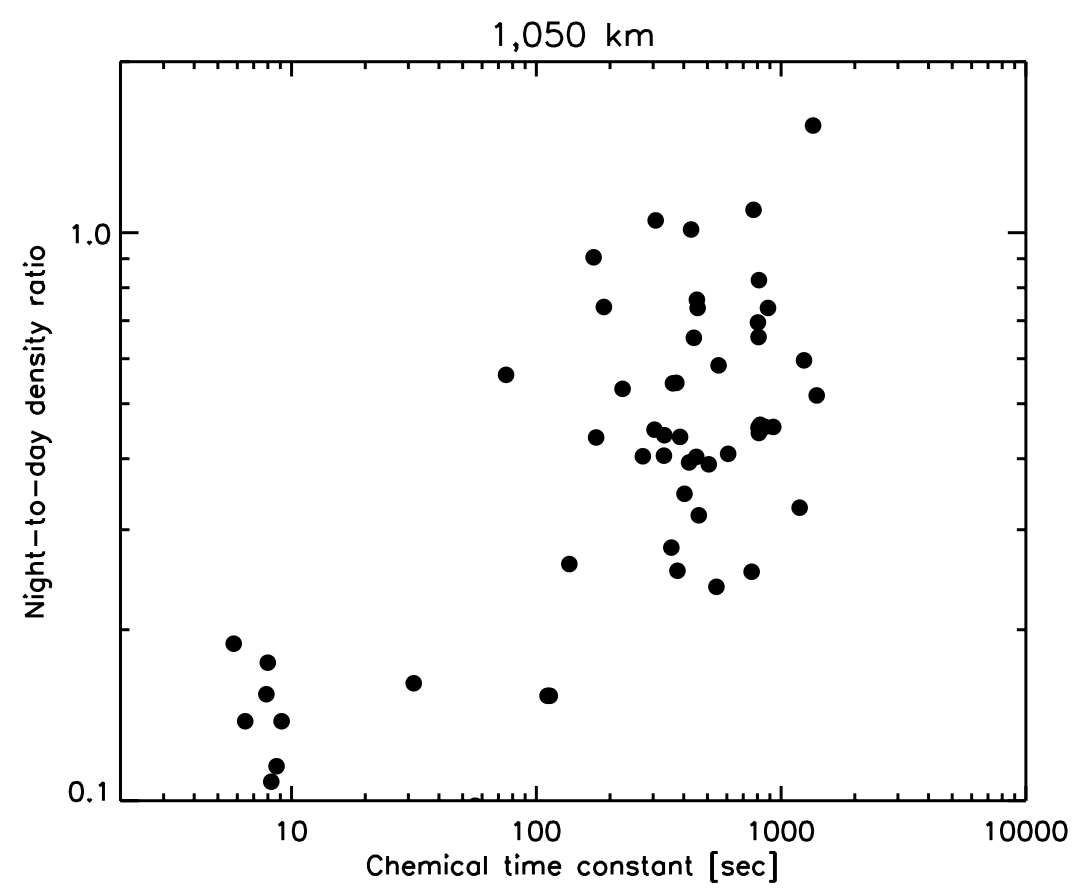

Figure 10. Observed night-to-day density ratio of all species identified in the INMS ion spectra as a function of their chemical lifetimes (or loss time constants). All ion species with observed dayside densities greater than $0.1 \mathrm{~cm}^{-3}$ are included. Figure 10 shows a clear correlation between the two quantities, demonstrating that the loss time constant is the key factor determining the diurnal variations in ionospheric densities.

dissociative recombination though it is a relatively light species [Vuitton et al., 2007].

[34] The fact that the observed ion densities are smaller near the morning terminator than near the evening terminator, as shown in Figure 5, supports the hypothesis that the nightside ionosphere is maintained by chemical survival of dayside ions. Ions observed near the evening terminator spend less time in darkness as compared with those near the morning terminator. Therefore the ion content at the evening terminator is expected to be less depleted than that at the morning terminator. However, it should be born in mind that such an observed feature needs to be verified with a larger INMS ion data set, especially because the ion distribution near the morning terminator relies exclusively on the outbound T32 data (see section 3). Above $\sim 1100 \mathrm{~km}$, the total ion densities near the morning terminator become higher than those on the nightside. This indicates that at relatively high altitudes, solar illumination starts to be an important ion source even near the terminator, as a result of the extended nature of Titan's atmosphere [Müller-Wodarg et al., 2000; Cravens et al., 2004; Agren et al., 2009].

[35] The importance of chemical survival of dayside ions in maintaining the nightside ionosphere depends on the relative time constants for chemistry and transport. A lower limit on the horizontal transport rate comes from Titan's solid body rotation period of $\sim 16$ days. This is significantly longer than the ion lifetimes given in Table 2, predicted from our time-dependent ion chemistry model (see section 5). Thus assuming solid body rotation and the lack of any nonsolar ionization sources on the nightside implies that the net ion density on the nightside should be much smaller than the measured value of $\sim 700 \mathrm{~cm}^{-3}$. To address this, we discuss below several mechanisms that may affect the ion distribution on the nightside of Titan.

[36] First, the significant ion/electron densities observed on Titan's nightside have been recognized by previous investigators who assumed that precipitation of energetic electrons from Saturn's magnetosphere were responsible for the phenomenon [Cravens et al., 2008b; Agren et al., 2009]. Electron precipitation can induce nightside ion spectra with similar characteristics to those observed. As an example, we show with the solid line in Figure 11 an ion spectrum on Titan's nightside calculated assuming ionization by $100 \mathrm{eV}$ electrons. The calculations are carried out for conditions appropriate to $1050 \mathrm{~km}$ in Titan's atmosphere and densities are calculated assuming steady state conditions. A net ionization rate of 0.03 ions $\mathrm{cm}^{-3} \mathrm{~s}^{-1}$ is used in the calculations, roughly $6 \%$ of the solar ionization rate at the same altitude for small SZAs. The ion spectrum calculated with a solar ionization rate of 5 ions $\mathrm{cm}^{-3} \mathrm{~s}^{-1}$ is given by the dotted line in Figure 11 for comparison. The total ion density implied by the electron precipitation model is $\sim 500 \mathrm{~cm}^{-3}$, roughly consistent with the INMS observations on Titan's nightside. The general shape of the spectrum associated with electron precipitation is also in agreement with that observed in that the density of short-lived ions is greatly reduced by more than an order of magnitude but the long-lived ions only by a factor of $2-3$. As with the timedependent situation, this is a consequence of the different loss mechanisms, i.e., "fast" loss through ion-neutral chemistry for short-lived ions and "slow" loss through electron dissociative recombination for long-lived ones. 


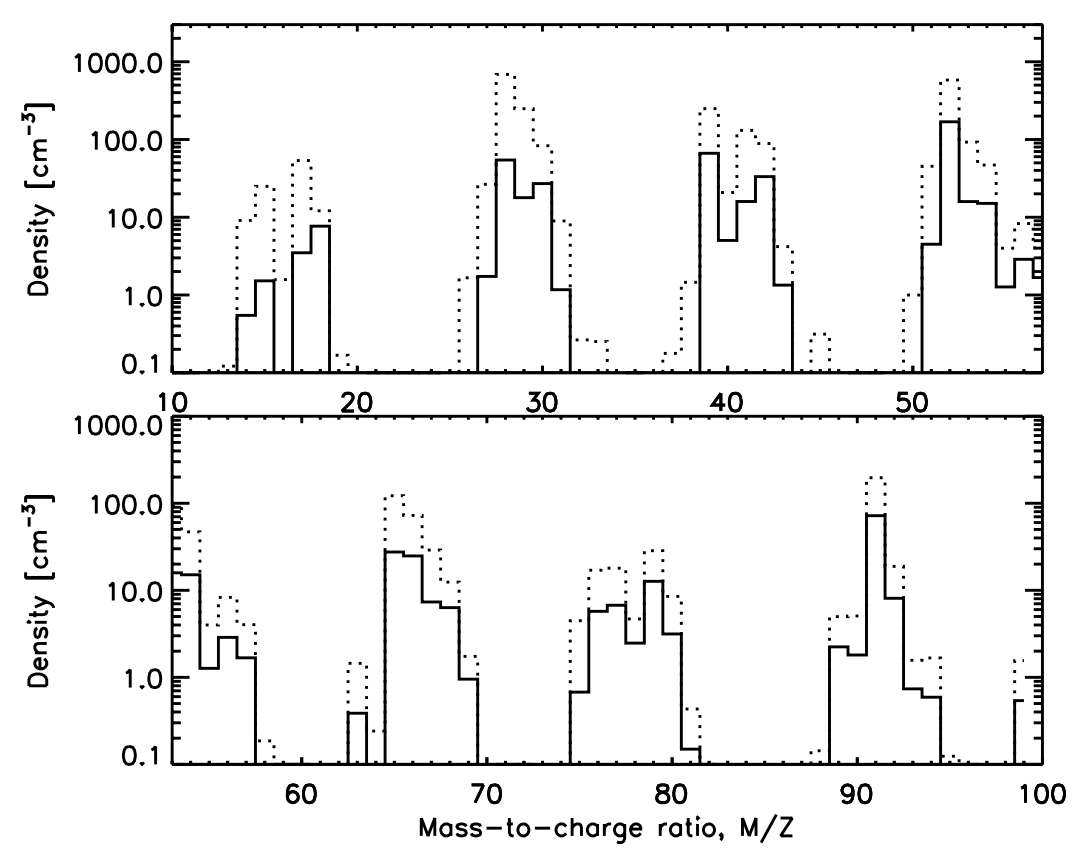

Figure 11. Ion mass spectra calculated with the solar model (dotted line) and the electron precipitation model (solid line) for an altitude of $1050 \mathrm{~km}$ in Titan's upper atmosphere. The electron precipitation model assumes a net ionization rate of 0.03 ions $\mathrm{cm}^{-3} \mathrm{~s}^{-1}$, about $6 \%$ of the adopted solar ionization rate. The total ion density of $\sim 500 \mathrm{~cm}^{-3}$ inferred from the electron precipitation model is roughly consistent with the observed value. The general shape of the observed nightside ion spectrum, as shown in Figure 4, is also reasonably reproduced by the electron precipitation model.

For this model, the lower electron density implies that the loss rates for the long-lived ions are reduced but the loss rates for the short-lived ions, primarily lost through ionneutral reactions, is unchanged. It should be noted that the short-lived ions in this electron precipitation model still have significant densities, though greatly reduced from the dayside values. As we show in the next section, timedependent models for the chemical survival of dayside ions predict far less density for short-lived ions at large solar illumination angles than that observed. The INMS observations of short-lived ions then imply the existence of some level of electron precipitation from Saturn's magnetosphere.

[37] Second, the actual day-to-night transport timescale depends on the dynamics of Titan's upper atmosphere, and as mentioned above, Titan's solid body rotation acts as an upper limit on the transport timescale. The dynamics of Titan's upper atmosphere are not well constrained, leading to uncertain transport rates. Despite this, it is clear that strong horizontal neutral winds could increase the transport rate and shorten the associated time constant considerably. Such horizontal neutral winds could be driven either by day-to-night temperature difference on Titan or by dynamical coupling with Titan's superrotating stratosphere [MüllerWodarg et al., 2000]. Existing Thermospheric Global Circulation Model (TGCM) calculations show that for solar minimum conditions (as suitable for the time when the INMS data were taken), the day-to-night temperature difference in Titan's upper atmosphere may drive horizontal neutral winds with speed up to $30 \mathrm{~m} \mathrm{~s}^{-1}$, in a pattern that blows from subsolar to antisolar and gives opposite wind speeds at the morning and evening terminators [Müller-
Wodarg et al., 2000]. However, the associated temperature difference of $\sim 10 \mathrm{~K}$ between Titan's dayside and nightside has not been confirmed by the present INMS neutral data [e.g., Müller-Wodarg et al., 2008; Cui et al., 2009] possibly because the limited sampling of the existing INMS data set. On the other hand, the actual wind speed might be significantly larger than that predicted from solar driven models if the dynamics in Titan's upper atmosphere is coupled with superrotation in the stratosphere [e.g., Flasar et al., 1981; Hubbard et al., 1993; Hourdin et al., 1995; Tokano et al., 1999]. In such cases, the thermospheric wind speed could reach several hundred $\mathrm{m} \mathrm{s}^{-1}$ in a circulating prograde pattern [Müller-Wodarg et al., 2000]. The notion of superrotating thermosphere is also supported by the observed latitudinal density variations on Titan [Müller-Wodarg et al., 2008]. In section 5, we show in some more detail how the diurnal variations of different ion species could be affected by the presence of a superrotating thermosphere on Titan. Such an effect is simulated by imposing enhanced solid body rotation rate in our time-dependent ion chemistry model.

[38] In this section, we present the interpretation for Titan's nightside ionosphere as a remnant of dayside ions. We also discuss the possibility that such an effect could be enhanced significantly by rapid horizontal neutral winds. While we understand that the chemical survival of dayside ions is always at work, at least because of the solid body rotation of Titan, it does not necessarily mean that the conventional scenario of electron precipitation from Saturn's magnetosphere is no longer valid. In this paper, we consider electron precipitation as well, and we show that the 
electron precipitation model can explain some features of the observed diurnal variations of Titan's ionosphere. Since the scenario of chemical survival of dayside ions has never been explored for Titan in previous works, we extend our investigation here with some numerical experiments based on a time-dependent ion chemistry model.

\section{Time-Dependent Ion Chemistry Model}

[39] We show in section 4 that several characteristics of the INMS ion data could be interpreted by the scenario of chemical survival of dayside ions to the nightside. This naturally explains the correlation between the observed night-to-day ion density ratios and the associated chemical time constants (as shown in Figure 10), as well as the observed asymmetry in the ion density profiles near the morning and evening terminators (as shown in Figure 5). In order to investigate this possible explanation, we need to make realistic estimates on chemical lifetimes and transport rates. We therefore constructed a time-dependent ion chemistry model for Titan's ionosphere to investigate the role of chemistry and transport in maintaining the nightside ionosphere. The results presented in this section cannot be regarded as a rigorous comparison between the model and data. On one hand, this is because the model and data are not identically sampled, and on the other hand, this is also because we do not have sufficient knowledge of the global circulation pattern in Titan's upper atmosphere, and thus day-to-night transport is only treated in an idealistic way (see below for details). Nevertheless, we show below that the general characteristics of the model results are similar to those seen in the INMS ion data, at least for long-lived ion species.

[40] Our time-dependent ion chemistry model includes a large network of 1641 chemical reactions [Vuitton et al., 2006, 2007, 2009], ionization by solar photons and suprathermal electrons (photoelectrons and their secondaries), and assumes no contribution from magnetospheric electrons. We include the effect of Titan's rotation to model the diurnal variations of the ionosphere. Such a rotation technique has been implemented recently by De La Haye et al. [2008]. The effect of horizontal winds is modeled in this work with enhanced rotation rate (see below for details). We also take into explicit account vertical transport through ambipolar diffusion, therefore local photochemical equilibrium $(\mathrm{PCE})$ is not a priori assumed in the model. Such vertical diffusion could be important at relatively high altitudes, where the chemical time constants become comparable to or greater than the diffusion timescales [e.g., $\mathrm{Ma}$ et al., 2006; Cravens et al., 2008b].

[41] The absorption rate of solar photons is calculated using the EUVAC solar spectrum (scaled to the location of Titan [Richards et al., 1994a, 1994b]) and the photoionization cross sections of $\mathrm{N}_{2}$ and $\mathrm{CH}_{4}$ from Samson et al. [1987, 1989] and Stolte et al. [1998]. Optical depth at any altitude is calculated by numerical integration along the line of sight to the Sun taking full account of the curved nature of the atmosphere, but assuming that the atmosphere can be treated locally as spherically symmetric. Energy degradation by suprathermal electrons is calculated with an energy grid of 200 bins from 0.1 to $10^{4} \mathrm{eV}$ with exponentially increas- ing bin width. We include all relevant energy loss processes in an $\mathrm{N}_{2}-\mathrm{CH}_{4}$ atmosphere (vibrational/rotational excitation, ionization and elastic collision [see Galand et al., 2006, and references therein]). The cross sections for electron impact ionization of $\mathrm{N}_{2}$ and $\mathrm{CH}_{4}$ are taken from Lummerzheim and Lilensten [1994], Davies et al. [1989] and Liu and Shemansky [2006]. In the calculations, we ignore the transport of suprathermal electrons and assume that the energy from electron impact of $\mathrm{N}_{2}$ and $\mathrm{CH}_{4}$ is deposited locally. Comparison with suprathermal electron transport calculations shows that this is an accurate assumption below $\sim 1300 \mathrm{~km}$ in terms of secondary electron production rate (M. Galand et al., Energy deposition in Titan's deep ionosphere, manuscript in preparation). At higher altitudes, direct photoionization dominates and the approximation is still accurate for the purpose of chemical calculations throughout the altitude range considered here (between 1000 and $1500 \mathrm{~km}$ ).

[42] The model for the neutral atmosphere used in the calculations is based on the work of Müller-Wodarg et al. [2008], Vuitton et al. [2006, 2007] and Cui et al. [2008, 2009]. The temperature profile and $\mathrm{N}_{2}$ and $\mathrm{CH}_{4}$ densities are taken directly from the empirical model of MüllerWodarg et al. [2008] for a latitude of $56^{\circ} \mathrm{N}$. This is also very nearly equal to the average of all INMS measurements in the northern hemisphere and thus represents average conditions for the observations considered here. The minor neutral species are based on the results of Vuitton et al. [2006, 2007] and Cui et al. [2009]. The Vuitton et al. [2006, 2007] analysis was based on a nightside ion spectrum (T5) assuming electron impact ionization, but we find that the neutral densities so derived also provide a good fit to the dayside ion spectrum, as shown by the solid line in Figure 3. We extend these results to full altitude profiles assuming that the species are distributed in diffusive equilibrium using the eddy diffusion profile of Yelle et al. [2008]. The only exception is $\mathrm{H}_{2}$, which is adopted directly from Cui et al. [2008].

[43] We use the same neutral model atmosphere for all local times in the models presented below. It is likely that there are some variations in atmospheric structure and densities with local time, though we do not yet have solid constraints on this [Cui et al., 2009]. In particular, the neutral densities derived by Vuitton et al. [2007] are based on T5 observations which occurred on the nightside of Titan, where conditions may be different from those on the dayside. Though many of the neutral species are characterized by long chemical time constants and may not exhibit strong day-to-night differences, some variations are still possible. The rationale for not including these variations is that the model results shown here are used in a strictly qualitative way. Given the lack of strong constraints on local time variations in neutral densities [Cui et al., 2009] and the qualitative nature of our goals for the model-data comparison, the complications and uncertainties introduced by an attempt to include diurnal variations in the neutral atmosphere are not warranted at this time.

[44] The model solves the time-dependent, coupled continuity equations of all ions

$$
\frac{\partial N_{i}}{\partial t}+\frac{1}{r^{2}} \frac{\partial}{\partial r}\left(r^{2} \Phi_{i}\right)=P_{i}-L_{i} N_{i}
$$




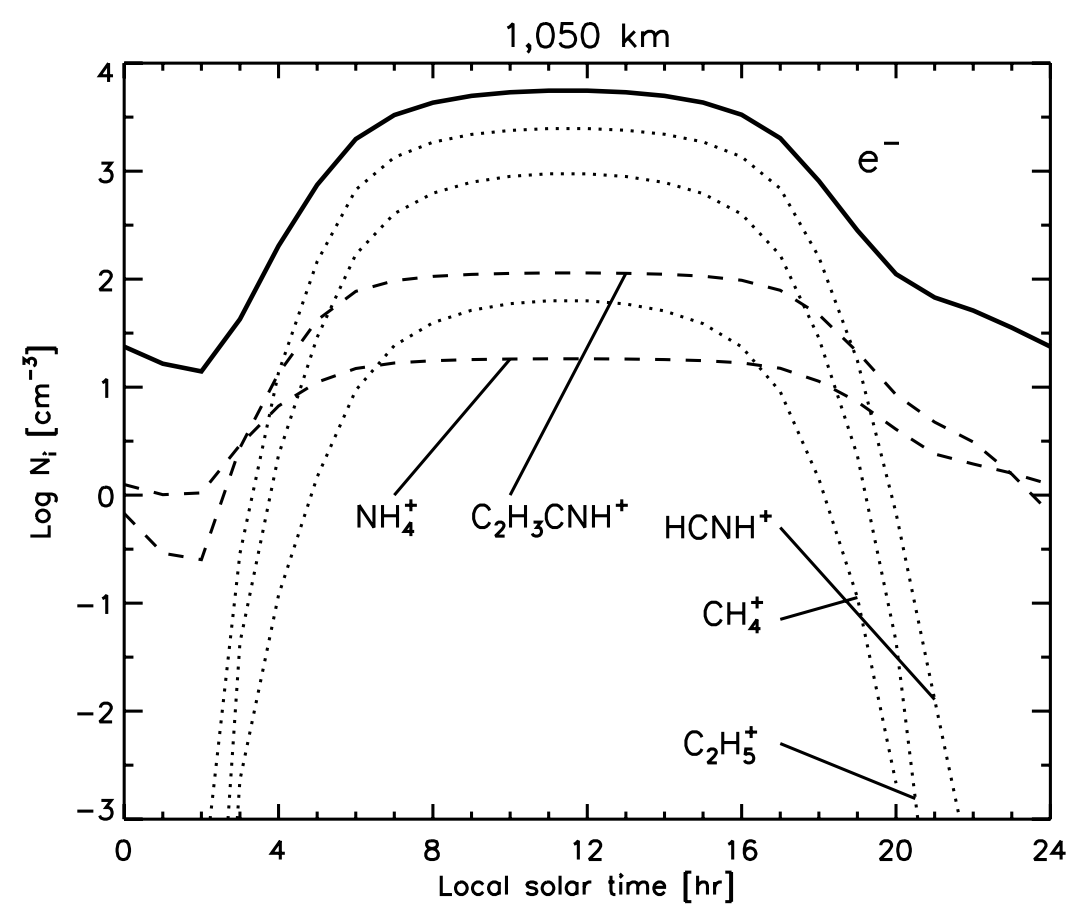

Figure 12. Modeled diurnal variations of electrons and several representative ion species at an altitude of $1050 \mathrm{~km}$. The electron densities are calculated as the total ion densities assuming quasi-neutrality. The variations of long-lived species $\left(\mathrm{NH}_{4}{ }^{+}\right.$and $\left.\mathrm{C}_{2} \mathrm{H}_{3} \mathrm{CNH}^{+}\right)$show clear effects of the chemical time constants with significant extension into the shadowed regions of the atmosphere.

where $N_{i}$ is the ion density, $r$ is the radius from the center of Titan, $P_{i}$ is the chemical production rate, $L_{i}$ is the chemical loss rate and the second term on the LHS represents the divergence of the flux, $\Phi_{i}$, given by

$$
\Phi_{i}=-D_{i}\left[\frac{\partial N_{i}}{\partial r}+N_{i}\left(\frac{1}{T_{i}} \frac{\partial T_{e}}{\partial r}+\frac{1}{H_{i}}+\frac{T_{e}}{T_{i}} \frac{1}{N_{e}} \frac{\partial N_{e}}{\partial r}\right)\right]
$$

where $H_{i}=\frac{k_{\mathrm{B}} T_{i}}{m_{i} g}$ is the ion scale height $\left(k_{\mathrm{B}}\right.$ is the Boltzmann constant, $g$ the local gravity, $m_{i}$ the ion mass), $T_{i}$ and $T_{e}$ are the ion and electron temperatures, $N_{e}=\Sigma_{i} N_{i}$ is the electron (or total ion) density and $D_{i}$ is the ion-neutral ambipolar diffusion coefficient. The term for electron density gradient enters equation (2) to remove the polarization electrostatic field that appears in both the electron and ion momentum equations [Schunk and Nagy, 2000]. We also assume equal ion and neutral temperatures throughout the model calculations. The chemical reactions in the model are based on the reaction list described by Vuitton et al. [2007] and Vuitton et al. [2009]. In equation (2), we have ignored the magnetic field term since for the typical magnetic field strength of $\sim 5 \mathrm{nT}$ in the vicinity of Titan [e.g., Backes et al., 2005], gyrofrequencies of ions in the mass range of 10-99 Da only become comparable with or greater than the ion-neutral collision frequencies above $\sim 1350 \mathrm{~km}$, while we are primarily interested in regions below (see section 2.1). Especially below the dayside ionization peak at $\sim 1120 \mathrm{~km}$, the ion-neutral collision frequencies, of order $1-10 \mathrm{~s}^{-1}$ depending on ion mass and altitude, are about two to three orders of magnitude greater than the gyrofrequencies, ensuring that the magnetic field has a minor effect on the ion distribution there. Ion densities are calculated by simultaneous solution of the coupled continuity equations including diurnally varying ionization rates, with a model resolution of $5 \mathrm{~km}$ in altitude and $600 \mathrm{sec}$ in local time. The calculations are run until the density variations at a particular local time vary from day to day by a negligible amount, usually $10^{-3} \mathrm{~cm}^{-3}$.

[45] Figure 12 shows the local time variations for several representative ion species at an altitude of $1050 \mathrm{~km}$, calculated from the time-dependent model described above. Only Titan's solid body rotation is included (i.e., assuming no horizontal neutral wind), and calculations are carried out at a latitude of $60^{\circ}$. The dotted and dashed lines in Figure 12 represent the diurnal variations of typical short-lived and long-lived species, respectively. The thick solid line gives the total ion density or electron density calculated from the model. Some interesting trends are immediately seen. The total ion densities (or electron densities) show asymmetric distribution about the local noon, with the densities at the evening terminator higher than the values at the morning terminator. This is consistent with that observed from the data (see Figure 5). The densities of short-lived ions, especially $\mathrm{CH}_{4}^{+}$, tend to follow the solar ionization rate, which is symmetric about local noon. The long-lived ions (e.g., $\mathrm{NH}_{4}^{+}$and $\mathrm{C}_{2} \mathrm{H}_{3} \mathrm{CNH}^{+}$) clearly show the effects of the chemical time constants with significant extension into the shadowed regions of the atmosphere. This is further illustrated in Figure 13 showing additional examples of longlived and heavy ions. Some of them display minimum densities near the local noon because of enhanced electron recombination rates on the dayside (e.g., $\mathrm{C}_{8} \mathrm{H}_{5}^{+}, \mathrm{C}_{8} \mathrm{H}_{7}^{+}$, $\mathrm{C}_{7} \mathrm{H}_{3} \mathrm{NH}^{+}$). The model calculations show that primarily in response to the time behavior of long-lived ions, the chemical survival of ions created on the dayside of Titan 


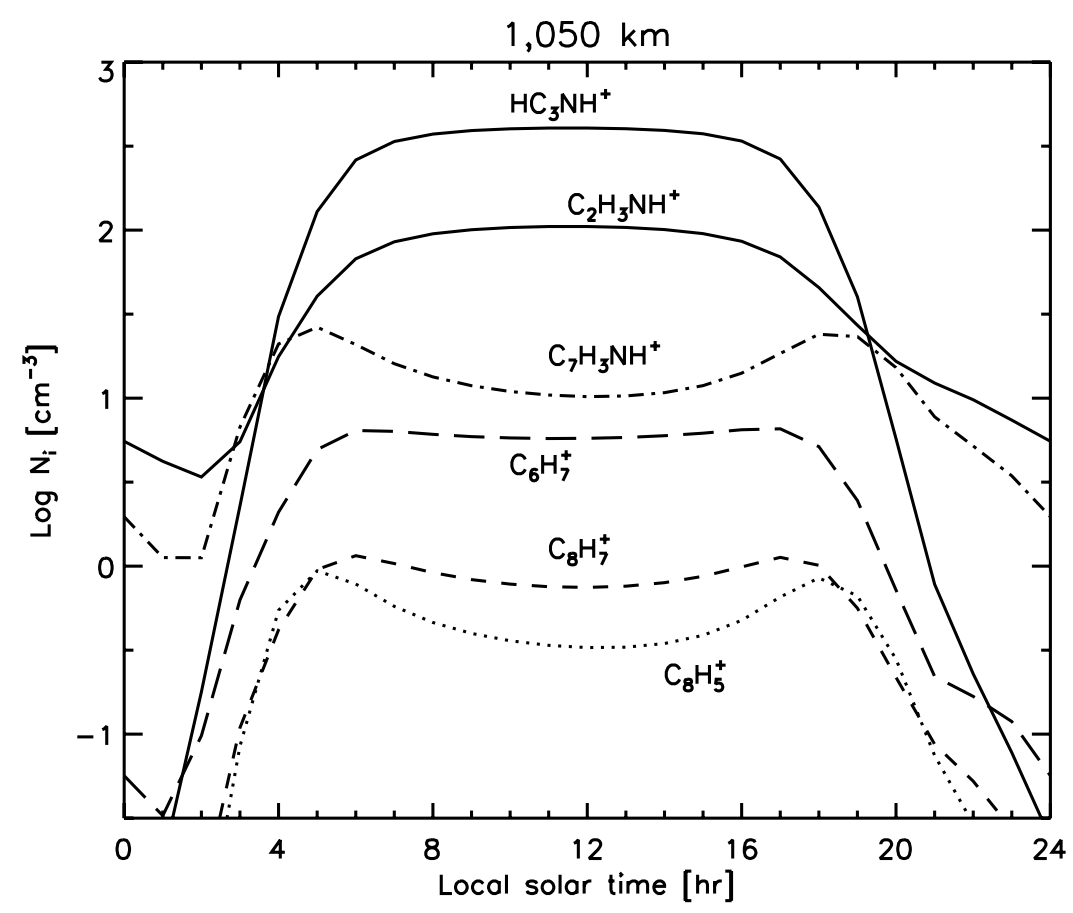

Figure 13. Modeled diurnal variations of several heavy ion species at an altitude of $1050 \mathrm{~km}$. Some species (e.g., $\mathrm{C}_{8} \mathrm{H}_{5}{ }^{+}, \mathrm{C}_{8} \mathrm{H}_{7}{ }^{+}$and $\mathrm{C}_{7} \mathrm{H}_{3} \mathrm{NH}^{+}$) display minimum densities near the local noon because of enhanced dissociative recombination rates on the dayside.

is able to maintain an appreciable ionosphere on the nightside, without taking into account impact ionization by precipitating electrons from Saturn's magnetosphere.

[46] The rapid disappearance of short-lived ions and persistence of long-lived ions, as revealed in Figures 12 and 13, reflect different time behaviors of these species. We can see this with a simple analytical approach. The time dependence of short-lived ions, which are lost primarily through ion-neutral chemistry, can be described by

$$
\frac{d N_{i}}{d_{t}}=-k_{i n} N_{n} N_{i}
$$

where $N_{i}$ and $N_{n}$ are the ion and neutral densities, and $k_{i n}$ is the corresponding reaction rate. $N_{n}$ is assumed to be constant over time (see above). This gives the solution to $N_{i}(t)$ as

$$
N_{i}(t)=N_{i}\left(t_{0}\right) \exp \left(-\frac{t-t_{0}}{t_{L}}\right)
$$

where $t_{0}$ is the initial time and $t_{L}=1 /\left(k_{i n} N_{n}\right)$ is the loss time constant (through ion-neutral chemistry). This implies that short-lived ions decay exponentially with time. On the other hand, the continuity equation for long-lived ions that are lost through recombination can be written as

$$
\frac{d N_{i}}{d_{t}}=-\alpha_{i} N_{i}^{2}
$$

where $\alpha_{i}$ is the recombination coefficient and for illustrative purpose, we assume that the atmosphere is composed of a single ion species with $N_{i}$ equal to the electron density. The solution to equation (5) is

$$
N_{i}(t)=N_{i}\left(t_{0}\right) /\left(1+\frac{t-t_{0}}{t_{L}}\right),
$$

where $t_{L}=1 /\left[\alpha_{i} N_{i}\left(t_{0}\right)\right]$ is the loss time constant (through recombination). The different time dependencies shown by equations (4) and (6) imply very different behaviors for loss through ion-neutral chemistry and loss through recombination. The density of ions that recombine with electrons decreases with time far more slowly than for ions that react with neutrals. After several chemical time constants into night, the former population is depleted by a factor of several while the latter disappear exponentially. It follows that the nightside ionosphere is composed primarily of ions that are lost through recombination.

[47] An additional and important effect that contributes to the diurnal variations in Titan's ionosphere is the production of heavy and long-lived ions well into darkness. As an example, Vuitton et al. [2007] showed that two important ion-neutral chemical pathways that lead to the loss of $\mathrm{HCNH}^{+}$are $\mathrm{HCNH}^{+}+\mathrm{HC}_{3} \mathrm{~N} \rightarrow \mathrm{HC}_{3} \mathrm{NH}^{+}+\mathrm{HCN}$ and $\mathrm{HCNH}^{+}+\mathrm{C}_{2} \mathrm{H}_{3} \mathrm{CN} \rightarrow \mathrm{C}_{2} \mathrm{H}_{3} \mathrm{CNH}^{+}+\mathrm{HCN}$. This implies that as Titan rotates into darkness, $\mathrm{HC}_{3} \mathrm{NH}^{+}$and $\mathrm{C}_{2} \mathrm{H}_{3} \mathrm{CNH}^{+}$ ions are produced at the expense of $\mathrm{HCNH}^{+}$loss. The ion lifetimes listed in Table 2 are estimated from $t_{\mathrm{L}} \approx N_{i} / L_{i}$ where $N_{i}$ is the ion density and $L_{i}$ is the total loss rate (in units of $\mathrm{cm}^{-3} \mathrm{~s}^{-1}$ ). As pointed out by Cravens et al. [2008b], for the so-called "terminal ions", the effective lifetimes, which depend on how far down the chemical chain these ions reside, could be significantly longer than 


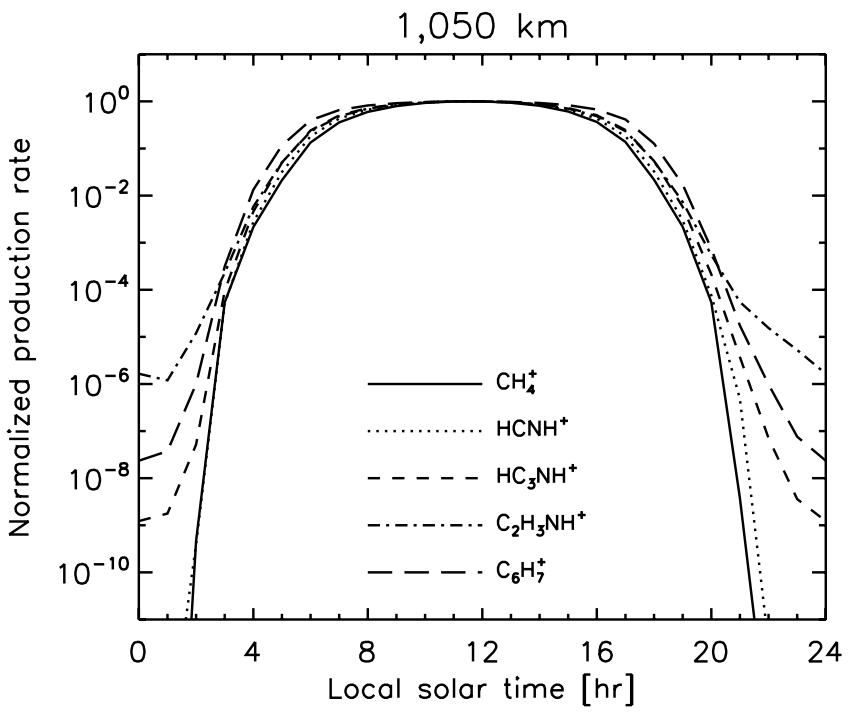

Figure 14. Normalized production rates of several representative ion species as a function of local solar time, calculated from the time-dependent ion chemistry model described in the text. The normalized production rate is defined as the ratio of the actual production rate to the production rate at local noon. The production rates of shortlived species such as $\mathrm{CH}_{4}{ }^{+}$and $\mathrm{HCNH}^{+}$decrease sharply as Titan rotates into darkness. However, for long-lived species such as $\mathrm{HC}_{3} \mathrm{NH}^{+}, \mathrm{C}_{2} \mathrm{H}_{3} \mathrm{NH}^{+}$, and $\mathrm{C}_{6} \mathrm{H}_{7}^{+}$, there are significant production rates on the nightside. the values inferred from $N_{i} / L_{i}$. The effect of nightside production of heavy ions is illustrated in Figure 14, which shows the normalized production rates (at $1050 \mathrm{~km}$ ) for several representative ion species as a function of local solar time, based on our time-dependent ion chemistry model. The normalized production rate is defined as the ratio of the actual production rate to the production rate at local noon. Figure 14 shows that for typical short-lived ions (e.g., $\mathrm{CH}_{4}^{+}$ and $\mathrm{HCNH}^{+}$), the production rates, which are roughly symmetric about the local noon, decrease sharply as Titan rotates into darkness. On the contrary, the production rates of long-lived ions (e.g., $\mathrm{HC}_{3} \mathrm{NH}^{+}, \mathrm{C}_{2} \mathrm{H}_{3} \mathrm{NH}^{+}$and $\mathrm{C}_{6} \mathrm{H}_{7}^{+}$) show a pattern that extends well into the nightside. These features are important for maintaining an appreciable nightside ionosphere on Titan, though they cannot be directly verified with the INMS ion data.

[48] In Figure 15, we show the altitude profiles of some representative ion species at local noon, midnight, dawn and dusk. These profiles are calculated from the time-dependent model for a latitude of $60^{\circ}$ and assuming solid body rotation only. Also shown in Figure 15 are the INMS ion densities averaged over the dayside (with SZA between $30^{\circ}$ and $70^{\circ}$ ) and nightside (with SZA beyond $120^{\circ}$ ) of Titan. The model profiles cannot be compared directly to observations because the observations do not sample the same constant local time and latitude. The comparison is nevertheless instructive. For long-lived ion species shown in Figure 15, the model profiles are in general agreement with the data, on both the dayside and nightside. However, for short-lived ions, the model predicts systematically lower densities than


Figure 15. Ion density profiles at different local solar times between 1000 and $1500 \mathrm{~km}$, calculated from the time-dependent ion chemistry model assuming solid body rotation only. Also shown are INMS ion densities averaged over Titan's dayside (black dots) and nightside (gray dots), respectively. The dayside densities correspond to average values in the SZA range of $30^{\circ}-70^{\circ}$, and the nightside densities correspond to average values in the SZA range of $120^{\circ}-180^{\circ}$. For the three short-lived species in the top, the model profiles at midnight are well below the selected density range and are thus not shown. 


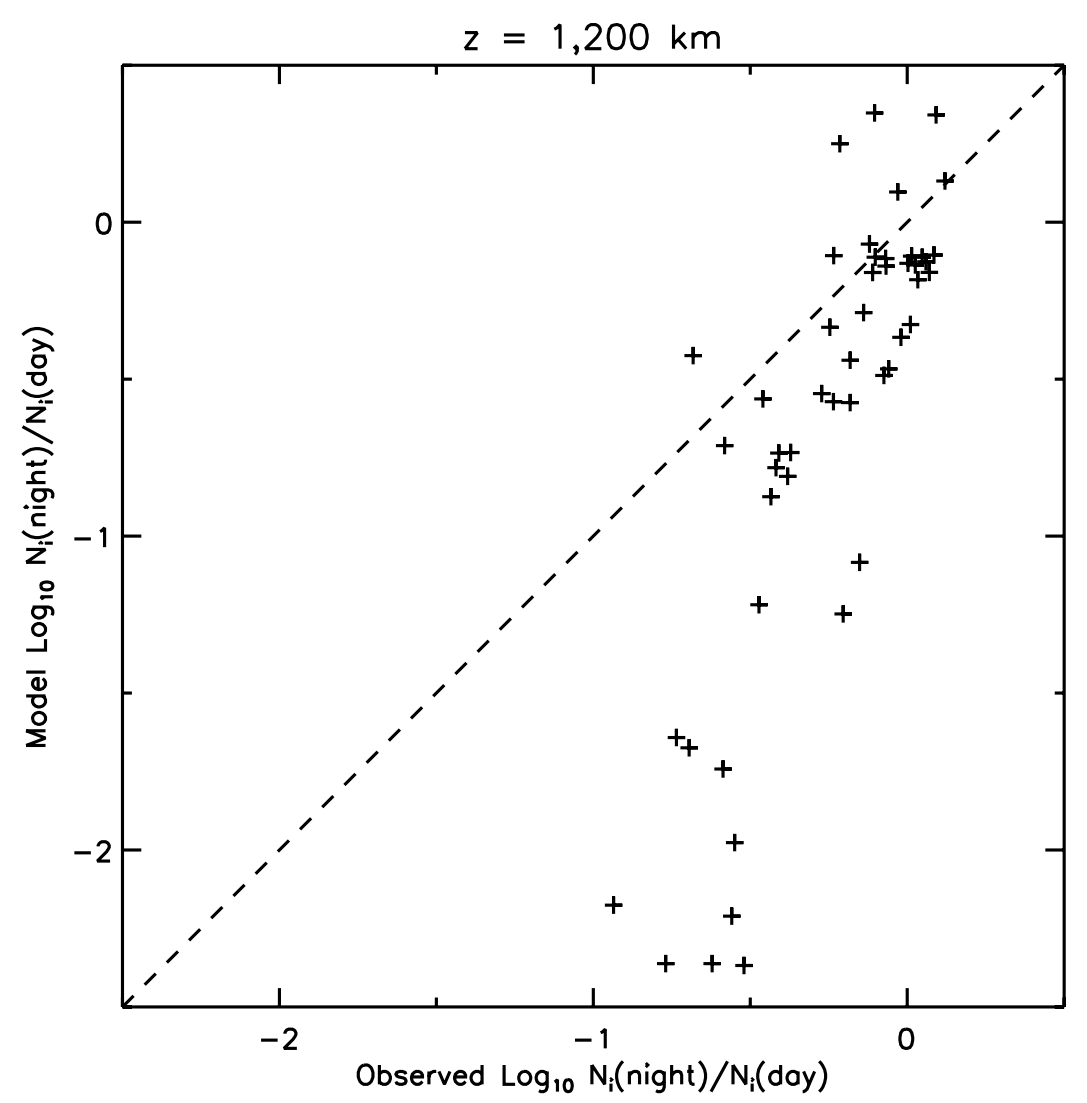

Figure 16. Night-to-day ion density ratios inferred from the time-dependent ion chemistry model, compared with the observed values at an altitude of $1200 \mathrm{~km}$. All ion species with observed dayside densities greater than $0.1 \mathrm{~cm}^{-3}$ are included. The dashed line shows equality between the observed and predicted density ratios.

the observations on the nightside, indicating that chemical survival from the dayside and associated with solid body rotation only accounts for a small fraction of the observed densities on the nightside for these ions.

[49] We show further in Figure 16 the night-to-day ion density ratios inferred from the time-dependent ion chemistry model, compared with the observed values at $1200 \mathrm{~km}$. The dashed line shows equality between the two quantities. The ratio is shown for all ion species with observed dayside densities greater than $0.1 \mathrm{~cm}^{-3}$. For typical long-lived species with modest depletion on the nightside, a general agreement between the observed and predicted night-to-day ratios is obtained, though with a significant scattering which could be associated with uncertainties in the ion reaction data. The scattering is also caused by the fact that the observations sample a range of latitude and local solar time while the model profiles are presented for fixed values of these parameters. However, for typical short-lived species with significant depletion on the nightside, there appears to be a systematic trend of underpredicted night-to-day density ratios, consistent with that seen in Figure 15. Such a systematic deviation cannot be interpreted by either the uncertainties in the ion reaction rates or the realistic sample distribution with respect to latitude and local solar time.

[50] The time-dependent model calculations taking into account Titan's solid body rotation clearly show persistence in the ion population from the dayside to the nightside. The general trend revealed by the model is consistent with the observed correlation between the loss time constants and night-to-day ion density ratios, as shown in Figure 10. However, the model-data comparison also shows that the predicted depletion for short-lived ions at low altitudes is significantly larger than that observed from the INMS data. To address such a deviation, we investigate further the effects of horizontal neutral winds. Since we do not yet have sufficient knowledge of circulation patterns in Titan's upper atmosphere [Müller-Wodarg et al., 2008], we adopt here a simplified scheme by imposing enhanced rotation rates in the time-dependent model. This effectively simulates a superrotating thermosphere. As discussed in section 4, (superrotating) horizontal neutral winds enhance the effect of ion survival from the dayside to the nightside, thus partly reducing the day-to-night difference in ion densities inferred from solid body rotation only.

[51] In Figure 17, we show the local time variation in total ion density (or electron density) calculated with the time-dependent ion chemistry model with an enhanced rotation speed of $100 \mathrm{~m} \mathrm{~s}^{-1}$ (black solid line), as compared with the case for solid body rotation only (gray solid line). Both calculations are carried out at a latitude of $60^{\circ}$. The solid body rotation rate at this latitude corresponds to a horizontal velocity of $9 \mathrm{~m} \mathrm{~s}^{-1}$. Figure 17 shows that with enhanced rotation speed, the diurnal variation of electron density is significantly reduced. We also show in Figure 17 the local time variations in the ion densities of two species, including $\mathrm{CH}_{5}^{+}$(as representative of a short-lived species, 


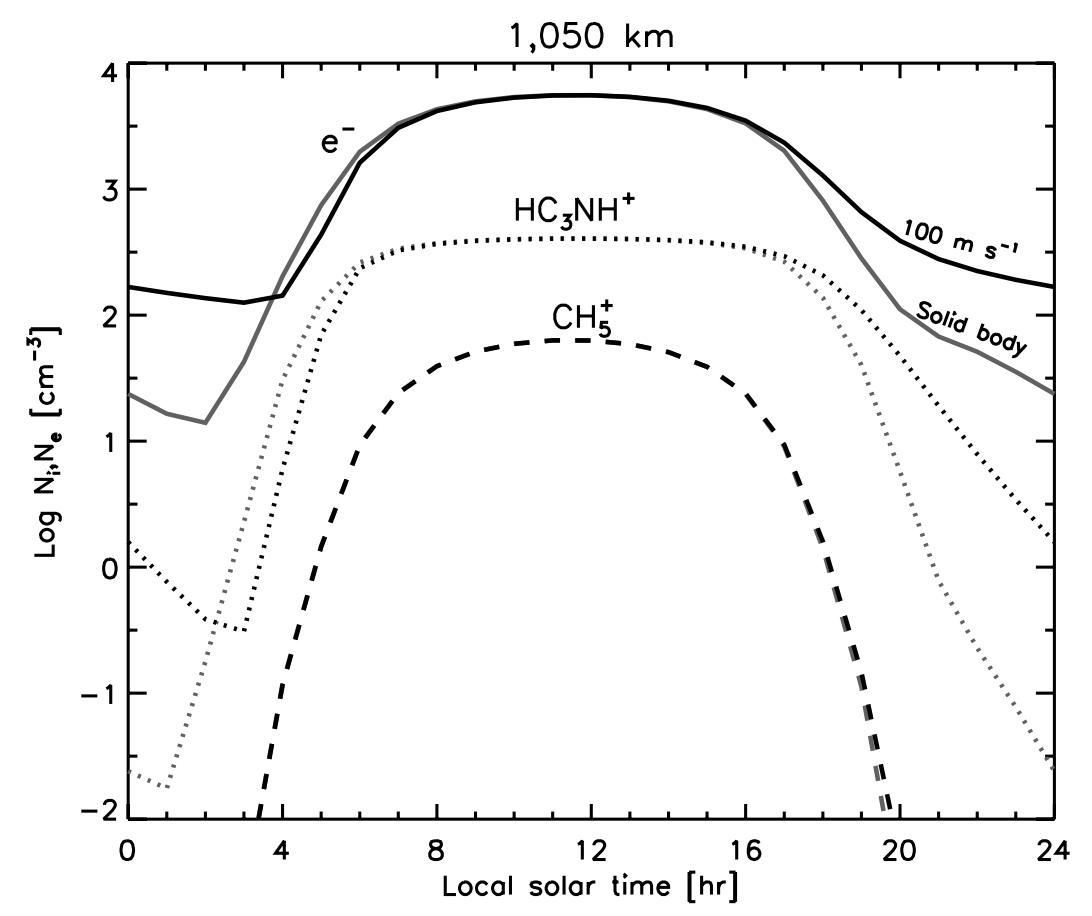

Figure 17. The local time variations of total ion (or electron) density (solid lines), $\mathrm{CH}_{5}{ }^{+}$density (dashed lines), and $\mathrm{HC}_{3} \mathrm{NH}^{+}$density (dotted lines), calculated with an enhanced rotation speed of $100 \mathrm{~m} \mathrm{~s}^{-1}$ (black lines) as compared with the case of solid body rotation only (gray lines). The enhanced rotation rate effectively simulates a superrotating horizontal wind in Titan's upper atmosphere, which reduces the day-to-night difference in ion densities.

see dotted lines) and $\mathrm{HC}_{3} \mathrm{NH}^{+}$(as representative of a longlived species, see dashed lines), with the black lines giving the results calculated with a wind speed of $100 \mathrm{~m} \mathrm{~s}^{-1}$ and the gray lines for the case of solid body rotation only.
Superrotation affects the diurnal variations of both light and heavy ion species, though the difference in the ion densities of light species between the two model runs cannot be seen on the density scale of Figure 17.
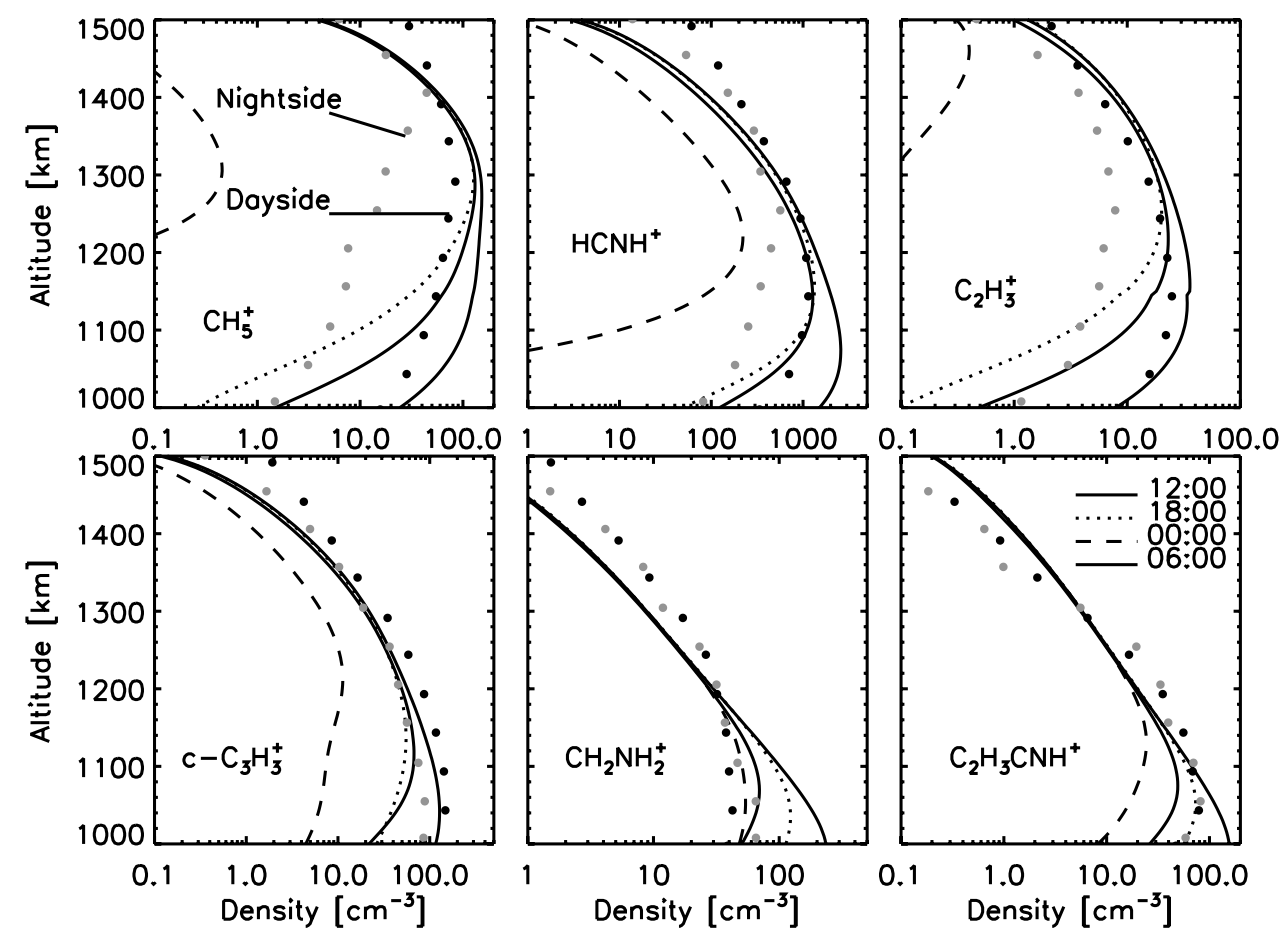

Figure 18. Same as Figure 15, except that an enhanced rotation speed of $100 \mathrm{~m} \mathrm{~s}^{-1}$ is adopted in the model calculations. 
[52] Similar to Figure 15, we show in Figure 18 the ion density profiles at different local solar times between 1000 and $1500 \mathrm{~km}$, calculated with the enhanced rotation rate of $100 \mathrm{~m} \mathrm{~s}^{-1}$. At local noon, dawn and dusk, the density profiles calculated with the enhanced rotation rate are similar to those obtained for the case of solid body rotation only. This implies that the ion densities at these local times are primarily controlled by solar illumination. As we have emphasized, the fact that solar illumination is still important near both the morning and evening terminators is because of the extended nature of Titan's atmosphere [Müller-Wodarg et al., 2000; Cravens et al., 2004; Agren et al., 2009]. On the other hand, at local midnight when photoionization is absent and chemical survival of dayside ions becomes important, the ion densities of all species shown in Figure 18 are significantly increased at all altitudes, as compared with the model profiles for solid body rotation (see Figure 15). The predicted altitude profiles of long-lived ions are in rough agreement with the INMS data. However, Figure 18 shows that even for the enhanced rotation rate, there is still clear discrepancy between the model and the data with respect to the nightside densities of short-lived species such as $\mathrm{CH}_{5}^{+}$. We expect that such a discrepancy becomes less significant when imposing an even larger rotation rate. However, we do not present here model calculations with horizontal wind speeds larger than $100 \mathrm{~m} \mathrm{~s}^{-1}$ since they are probably unrealistic [e.g., Müller-Wodarg et al., 2000, 2008]. Instead, the significant densities of short-lived ions observed on Titan's nightside is more likely to be an indicator of impact ionization by precipitating electrons from Saturn's magnetosphere, as mentioned in section 4 . We will also attempt in a later work more rigorous modeling of the diurnal variations of Titan's ionosphere by adopting realistic general circulation patterns of neutral winds on Titan [e.g., MüllerWodarg et al., 2000, 2008]. The downwelling and upwelling motions associated with such wind patterns may redistribute both short-lived and long-lived ions significantly.

\section{Discussions and Conclusions}

[53] In this paper, we present an analysis of the diurnal variations of Titan's ionosphere based on a sample of INMS/OSI data obtained during 8 Cassini flybys with the satellite. Though the INMS data show a general trend of ion depletion well beyond the terminator, the total ion densities on the nightside are still appreciable, characterized by a plateau at a level of $\sim 700 \mathrm{~cm}^{-3}$ below $\sim 1300 \mathrm{~km}$. There is clear asymmetry between the observed ion density profiles near the morning and evening terminators, in that the ion densities at dawn are significantly lower than at dusk. Our analysis reveals clear distinctions between the ionospheric structures of light and heavy ions in that light ions show strong depletion on the nightside whereas the depletion of heavy ions is less pronounced. The observed density plateau in the INMS total ion density distribution is the combined result of the distinct nightside distributions of light and heavy ions. The observed night-to-day ion density ratio is found to be strongly correlated with ion lifetime.

[54] The above observations suggest that the chemical survival of dayside ions provides an important ion source for the nightside of Titan. The distinctions between the diurnal variations of light and heavy ions are associated with their different chemical loss pathways, in that light ions are primarily lost through ion-neutral chemistry (with relatively small chemical time constants), while heavy ones are primarily lost through electron dissociative recombination (with relatively large recombination time constants). The observed diurnal variation of the apparent exception, $\mathrm{NH}_{4}^{+}$, can also be interpreted by such a scenario, since $\mathrm{NH}_{4}^{+}$is primarily lost through recombination though it is a light species. We have constructed a time-dependent ion chemistry model to investigate the effects of chemistry and transport on the ionospheric structures of Titan for the case of solid body rotation and the case with superrotating horizontal winds. The general characteristics of the observed diurnal variations are well captured by the model. The model with superrotating winds presents significantly more ions on the nightside, as compared with the case of solid body rotation only. However, in both cases the predicted densities of short-lived ions (e.g., $\mathrm{CH}_{5}^{+}$) are systematically lower than the observations on the nightside.

[55] The discrepancy between the model and data with respect to the nightside distribution of short-lived ions could be interpreted by electron precipitation from Saturn's magnetosphere. Both precipitation of magnetospheric electrons and ion survival from the dayside can contribute to Titan's nightside ionosphere and it is difficult to disentangle the contributions of these two sources. There are several powerful arguments for the importance of electron precipitation. The significant densities of very short-lived ions (e.g., $\mathrm{CH}_{3}^{+}$, $\mathrm{N}^{+}$) on the nightside strongly suggests electron precipitation because it seems unlikely that these species could survive from the dayside. We have also shown that model calculations based on electron precipitation can produce a nightside ion spectrum in rough agreement with the INMS observations. Moreover, the correlations between electron flux and $\mathrm{CH}_{5}^{+}$ion density demonstrated by Cravens et al. [2008b] shows that, at least for that encounter, electron impact ionization was occurring on the nightside. However, it is dangerous to generalize this conclusion because the magnetospheric electron and ion fluxes measured during T5 are far higher than for any other encounter [Cravens et al., $2008 \mathrm{~b}$ ]. One of the difficulties with assessing the importance of magnetospheric precipitation is our lack of knowledge of the magnetic topology in the vicinity of Titan. Theoretical predictions of $\mathbf{B}$ field penetration into the ionosphere are not yet precise enough to predict at which locations magnetospheric precipitation is important. Thus though it is possible to explain much of the ionospheric data with models that incorporate electron precipitation, it is unclear if agreement between models and data reflect the free parameters that the models must employ or the underlying physical reality. Finally, we note that electron precipitation does not necessarily imply a connection with Saturn's magnetosphere because it is possible that some regions on the nightside of Titan are magnetically connected to the dayside and precipitating electrons could simply be suprathermal electrons from the dayside. Better knowledge of the $\mathbf{B}$ field topology is required to investigate this possibility.

[56] Several characteristics of the observed ion densities argue for the importance of chemical survival through dayto-night transport, enhanced by superrotating horizontal winds. The different diurnal variations seen in short-lived 
and long-lived species, especially the strong correlation between the observed night-to-day ion density ratios and the associated ion lifetimes, are naturally explained by such a scenario. The observed asymmetry between the dawn and dusk ion density profiles also strongly supports this interpretation. Time-dependent model calculations show that the long-lived ions can survive well into the nightside, with the observed night-to-day density ratio of $\sim 2$ naturally explained as a slow decay of density with local time. For the model calculations with superrotating horizontal winds, the good agreement between the calculated and observed altitude profiles for long-lived ions on both the dayside and nightside of Titan is rather compelling since there is only one free parameter involved.

[57] Investigation into the nature of Titan's nightside ionosphere can be extended in several ways. Collection of more data will certainly help, not only by further characterizing the variations with SZA, but also by enabling measurement of variations with other geophysical variables, such as longitude or magnetospheric interaction geometry (J. G. Luhmann et al., Magnetospheric plasma interaction effects on Titan's ionosphere, manuscript in preparation). Accurate models for the B field topology in the vicinity of Titan would help enormously by permitting well constrained theoretical predictions for the patterns and levels of magnetospheric precipitation. Finally, simulations of the nightside ionosphere based on realistic general circulation models for neutral winds would allow more accurate estimation of the effects of chemical survival through day-tonight transport. We expect that the downwelling and upwelling motions associated with such models produce more complicated effects than predicted by the numerical experiments done so far. This also implies that with a better understanding of the ion chemistry on Titan as well as a better constraint on the contribution of electron precipitation, it may be possible to use the observed pattern of diurnal variations in Titan's ionosphere to constrain the wind field.

[58] Acknowledgments. J.C. and M.G. are supported by the Science and Technology Facilities Council (STFC) rolling grant to Imperial College London. R.V.Y. and P.P.L. acknowledge the support from NASA through grant NAG5-12699 to the University of Arizona and subcontract 699083KC from the Southwest Research Institute. I.M.-W. is funded by a University Research Fellowship of the British Royal Society. J.H.W. is funded by NASA and the Jet Propulsion Laboratory contract 1283095 with Southwest Research Institute.

[59] Wolfgang Baumjohann thanks Janet Luhmann and Darrell Strobel for their assistance in evaluating this paper.

\section{References}

Ågren, K., et al. (2007), On magnetospheric electron impact ionization and dynamics in Titan's ram-side and polar ionosphere-A Cassini case study, Ann. Geophys., 25, 2359-2369.

Ågren, K., J.-E. Wahlund, P. Garnier, R. Modolo, J. Cui, M. Galand, and I. C. F. Müller-Wodarg (2009), The ionospheric structure of Titan, Planet. Space Sci., doi:10.1016/j.pss.2009.04.012, in press.

Backes, H., et al. (2005), Titan's magnetic field signature during the first Cassini encounter, Science, 308, 992-995, doi:10.1126/science.1109763.

Bird, M. K., R. Dutta-Roy, S. W. Asmar, and T. A. Rebold (1997), Detection of Titan's ionosphere from Voyager 1 radio occultation observations, Icarus, 130, 426-436, doi:10.1006/icar.1997.5831.

Carrasco, N., C. Alcaraz, O. Dutuit, S. Plessis, R. Thissen, V. Vuitton, R. V. Yelle, and P. Pernot (2008), Sensitivity of a Titan ionospheric model to the ion-molecule reaction parameters, Planet. Space Sci., 56, 16441657, doi:10.1016/j.pss.2008.04.007.
Coates, A. J., F. J. Crary, G. R. Lewis, D. T. Young, J. H. Waite Jr., and E. C. Sittler Jr. (2007), Discovery of heavy negative ions in Titan's ionosphere, Geophys. Res. Lett., 34, L22103, doi:10.1029/2007GL030978.

Cravens, T. E., J. Vann, J. Clark, J. Yu, C. N. Keller, and C. Brull (2004), The ionosphere of Titan: an updated theoretical model, Adv. Space Res., 33, 212-215, doi:10.1016/j.asr.2003.02.012.

Cravens, T. E., et al. (2005), Titan's ionosphere: Model comparisons with Cassini Ta data, Geophys. Res. Lett., 32, L12108, doi:10.1029/ 2005 GL023249.

Cravens, T. E., et al. (2006), Composition of Titan's ionosphere, Geophys. Res. Lett., 33, L07105, doi:10.1029/2005GL025575.

Cravens, T. E., I. P. Robertson, S. A. Ledvina, D. Mitchell, S. M. Krimigis, and J. H. Waite Jr. (2008a), Energetic ion precipitation at Titan, Geophys. Res. Lett., 35, L03103, doi:10.1029/2007GL032451.

Cravens, T. E., et al. (2008b), Model-data comparisons for Titans nightside ionosphere, Icarus, 199, 174-188, doi:10.1016/j.icarus.2008.09.005.

Cui, J., R. V. Yelle, and K. Volk (2008), Distribution and escape of molecular hydrogen in Titan's thermosphere and exosphere, J. Geophys. Res., 113, E10004, doi:10.1029/2007JE003032.

Cui, J., et al. (2009), Analysis of Titan's neutral upper atmosphere from Cassini Ion Neutral Mass Spectrometer measurements, Icarus, 200, 581 615, doi:10.1016/j.icarus.2008.12.005.

Davies, D. K., L. E. Kline, and W. E. Bies (1989), Measurements of swarm parameters and derived electron collision cross sections in methane, J. Appl. Phys., 65, 3311-3323, doi:10.1063/1.342642.

De La Haye, V., J. H. Waite Jr., T. E. Cravens, I. P. Robertson, and S. Lebonnois (2008), Coupled ion and neutral rotating model of Titan's upper atmosphere, Icarus, 197, 110-136, doi:10.1016/j.icarus. 2008.03.022.

Flasar, F. M., R. E. Samuelson, and B. J. Conrath (1981), Titan's atmosphere-Temperature and dynamics, Nature, 292, 693-698, doi:10.1038/292693a0.

Fox, J. L., and R. V. Yelle (1997), Hydrocarbon ions in the ionosphere of Titan, Geophys. Res. Lett., 24, 2179-2182.

English, M. A., L. M. Lara, R. D. Lorenz, P. R. Ratcliff, and R. Rodrigo (1996), Ablation and chemistry of meteoric materials in the atmosphere of Titan, Adv. Space Res., 17, 157-160, doi:10.1016/02731177(95)00774-9.

Galand, M., J. Lilensten, D. Toublanc, and S. Maurice (1999), The ionosphere of Titan: Ideal diurnal and nocturnal cases, Icarus, 140, 92-105, doi:10.1006/icar.1999.6113

Galand, M., R. V. Yelle, A. J. Coates, H. Backes, and J.-E. Wahlund (2006), Electron temperature of Titan's sunlit ionosphere, Geophys. Res. Lett., 33, L21101, doi:10.1029/2006GL027488.

Gan, L., C. N. Keller, and T. E. Cravens (1992), Electrons in the ionosphere of Titan, J. Geophys. Res., 97, 12,137-12,151.

Hourdin, F., O. Talagrand, R. Sadourny, R. Courtin, D. Gautier, and C. P. McKay (1995), Numerical simulation of the general circulation of the atmosphere of Titan, Icarus, 117, 358-374, doi:10.1006/icar.1995.1162.

Hubbard, W. B., et al. (1993), The occultation of 28 SGR by Titan, Astron. Astrophys., 269, 541-563.

Ip, W.-H. (1990), Meteoroid ablation processes in Titan's atmosphere, Nature, 345, 511-512, doi:10.1038/345511a0

Keller, C. N., and T. E. Cravens (1994), One-dimensional multispecies hydrodynamic models of the wakeside ionosphere of Titan, J. Geophys. Res., 99, 6527-6536.

Keller, C. N., T. E. Cravens, and L. Gan (1992), A model of the ionosphere of Titan, J. Geophys. Res., 97, 12,117-12,135.

Keller, C. N., T. E. Cravens, and L. Gan (1994), One-dimensional multispecies magnetohydrodynamic models of the ramside ionosphere of Titan, J. Geophys. Res., 99, 6511-6525.

Keller, C. N., V. G. Anicich, and T. E. Cravens (1998), Model of Titans ionosphere with detailed hydrocarbon ion chemistry, Planet. Space Sci., $46,1157-1174$

Kliore, A. J., et al. (2008), First results from the Cassini radio occultations of the Titan ionosphere, J. Geophys. Res., 113, A09317, doi:10.1029/ 2007JA012965.

Liu, X., and D. E. Shemansky (2006), Analysis of electron impact ionization properties of methane, J. Geophys. Res., 111, A04303, doi:10.1029/ 2005JA011454.

López-Moreno, J. J., et al. (2008), Structure of Titan's low altitude ionized layer from the Relaxation Probe onboard HUYGENS, Geophys. Res. Lett., 35, L22104, doi:10.1029/2008GL035338.

Lummerzheim, D., and J. Lilensten (1994), Electron transport and energy degradation in the ionosphere: Evaluation of the numerical solution, comparison with laboratory experiments and auroral observations, Ann. Geophys., 12, 1039-1051, doi:10.1007/s005850050127.

Ma, Y.-J., A. F. Nagy, T. E. Cravens, I. V. Sokolov, K. C. Hansen, J.-E. Wahlund, F. J. Crary, A. J. Coates, and M. K. Dougherty (2006), Com- 
parisons between MHD model calculations and observations of Cassini flybys of Titan, J. Geophys. Res., 111, A05207, doi:10.1029/ 2005JA011481.

McLain, J. L., V. Poterya, C. D. Molek, N. G. Adams, and L. M. Babcock (2004), Flowing afterglow studies of the temperature dependencies for dissociative recombination of $\mathrm{O}_{2}^{+}, \mathrm{CH}_{5}^{+}, \mathrm{C}_{2} \mathrm{H}_{5}^{+}$and $\mathrm{C}_{6} \mathrm{H}_{7}^{+}$with electrons, J. Phys. Chem. A, 108, 6704-6708, doi:10.1021/jp0402151.

Molina-Cuberos, G. J., H. Lammer, W. Stumptner, K. Schwingenschuh, H. O. Rucker, J. J. López-Moreno, R. Rodrigo, and T. Tokano (2001), Ionospheric layer induced by meteoric ionization in Titan's atmosphere, Planet. Space Sci., 49, 143-153, doi:10.1016/S0032-0633(00)00133-1.

Müller-Wodarg, I. C. F., R. V. Yelle, M. Mendillo, L. A. Young, and A. D Aylward (2000), The thermosphere of Titan simulated by a global threedimensional time-dependent model, J. Geophys. Res., 105, 20,83320,856 .

Müller-Wodarg, I. C. F., R. V. Yelle, J. Cui, and J. H. Waite Jr. (2008), Horizontal structures and dynamics of Titan's thermosphere, J. Geophys. Res., 113, E10005, doi:10.1029/2007JE003033.

Nagy, A. F., and T. E. Cravens (1998), Titans ionosphere: A review, Planet. Space Sci., 46, 1149-1155.

Richards, P. C., J. A. Fennelly, and D. G. Torr (1994a), EUVAC: A solar EUV flux model for aeronomic calculations, J. Geophys. Res., 99, $8981-$ 8992.

Richards, P. C., J. A. Fennelly, and D. G. Torr (1994b), Correction to "EUVAC: A solar EUV flux model for aeronomic calculations", J. Geophys. Res., 99, 13,283.

Roboz, A., and A. F. Nagy (1994), The energetics of Titan's ionosphere, J. Geophys. Res., 99, 2087-2093.

Samson, J. A. R., T. Masuoka, P. N. Pareek, and G. C. Angel (1987), Total and dissociative photoionization cross sections of $\mathrm{N}_{2}$ from threshold to 107 eV, J. Chem. Phys., 86, 6128-6132, doi:10.1063/1.452452.

Samson, J. A. R., G. N. Haddad, T. Masuoka, P. N. Pareek, and D. A. L. Kilcoyne (1989), Ionization yields, total absorption, and dissociative photoionization cross sections of $\mathrm{CH}_{4}$ from 110-950 ̊, J. Chem. Phys., 90, 6925-6932, doi:10.1063/1.456267.

Schunk, R. W., and A. F. Nagy (2000), Ionospheres: physics, plasma physics, and chemistry, in Cambridge Atmos. Space Sci. Ser., edited by A. J. Dressler, J. T. Houghton, and M. J. Rycroft, 117 pp., Cambridge Univ. Press, Cambridge, U. K.

Stolte, W. C., Z. X. He, J. N. Cutler, Y. Lu, and J. A. R. Samson (1998), Dissociative photoionization cross sections of $\mathrm{N}_{2}$ and $\mathrm{O}_{2}$ from 100 to 800 eV, At. Data Nucl. Data Tables, 69, 171-179.

Tokano, T., F. M. Neubauer, M. Laube, and C. P. McKay (1999), Seasonal variation of Titans atmospheric structure simulated by a general circulation model, Planet. Space Sci., 47, 493-520.

Vuitton, V., R. V. Yelle, and V. G. Anicich (2006), The nitrogen chemistry of Titan's upper atmosphere revealed, Astrophys. J., 647, L175-L178, doi:10.1086/507467.
Vuitton, V., R. V. Yelle, and M. J. McEwan (2007), Ion chemistry and $\mathrm{N}$-containing molecules in Titan's upper atmosphere, Icarus, 191, 722 742, doi:10.1016/j.icarus.2007.06.023.

Vuitton, V., P. P. Lavvas, R. V. Yelle, M. Galand, A. Wellbrock, G. R. Lewis, A. J. Coates, and J.-E. Wahlund (2009), Negative ion chemistry in Titan's upper atmosphere, Planet. Space Sci., doi:10.1016/ j.pss.2009.04.004, in press.

Wahlund, J.-E., et al. (2005), Cassini measurements of cold plasma in the ionosphere of Titan, Science, 308, 986, doi:10.1126/science.1109807.

Waite, J. H., Jr., et al. (2004), The Cassini Ion and Neutral Mass Spectrometer (INMS) investigation, Space Sci. Rev., 114, 113-231, doi:10.1007/ s11214-004-1408-2.

Waite, J. H., Jr., et al. (2005), Ion neutral mass spectrometer results from the first flyby of Titan, Science, 308, 982-986, doi:10.1126/science. 1110652.

Waite, J. H., Jr., D. T. Young, T. E. Cravens, A. J. Coates, F. J. Crary, B. Magee, and J. Westlake (2007), The process of tholin formation in Titans upper atmosphere, Science, 316, 870-875, doi:10.1126/ science. 1139727

Yelle, R. V., N. Borggren, V. de La Haye, W. T. Kasprzak, H. B. Niemann, I. C. F. Müller-Wodarg, and J. H. Waite Jr. (2006), The vertical structure of Titan's upper atmosphere from Cassini Ion Neutral Mass Spectrometer measurements, Icarus, 182, 567-576, doi:10.1016/j.icarus.2005.10.029.

Yelle, R. V., J. Cui, and I. C. F. Müller-Wodarg (2008), Methane escape from Titan's atmosphere, J. Geophys. Res., 113, E10003, doi:10.1029/ 2007JE003031.

T. E. Cravens, Department of Physics and Astronomy, University of Kansas, Lawrence, KS 66045, USA.

J. Cui, M. Galand, and I. C. F. Müller-Wodarg, Space and Atmospheric Physics Group, Department of Physics, Imperial College, Prince Consort Road, London SW7 2BW, UK. (j.cui@imperial.ac.uk)

W. T. Kasprzak, Solar System Exploration Division, NASA Goddard Space Flight Center, Greenbelt, MD 20771, USA.

P. P. Lavvas and R. V. Yelle, Lunar and Planetary Laboratory, University of Arizona, 1629 East University Boulevard, Tucson, AZ 85721, USA.

V. Vuitton, Laboratoire de Planétologie de Grenoble, Université Joseph Fourier/CNRS, F-38041 Grenoble, France.

J.-E. Wahlund, Uppsala Division, Swedish Institute of Space Physics, Box 537, SE-751 21 Uppsala, Sweden.

J. H. Waite Jr., Space Science and Engineering Division, Southwest Research Institute, 6220 Culebra Road, San Antonio, TX 78238, USA. 